\title{
Anthraquinone laxative-altered gut microbiota induces colonic mucosal barrier dysfunction for colorectal cancer progression
}

\section{Zhonghong Wei}

Nanjing University of Chinese Medicine

Jiawei Wu

Nanjing University of Chinese Medicine

Yu Yang

Nanjing University of Chinese Medicine

peiliang Shen

Nanjing University of Chinese Medicine

\section{Peng Cheng}

Nanjing University of Chinese Medicine

Li Tao

Yangzhou University

\section{Yunlong Shan}

China Pharmaceutical University

\section{zhiguang Sun}

Nanjing University of Chinese Medicine

Yin Lu ( $\sim$ luyingreen@njucm.edu.cn )

Nanjing University of Chinese Medicine https://orcid.org/0000-0003-2063-8485

\section{Research}

Keywords: Anthraquinone laxative, sennoside A, gut microbiota, Akkermansia muciniphila, colonic mucosal barrier, colitis-associated colon cancer

Posted Date: October 20th, 2020

DOl: https://doi.org/10.21203/rs.3.rs-72368/v2

License: (c) (1) This work is licensed under a Creative Commons Attribution 4.0 International License.

Read Full License 


\section{Abstract}

Background: The safety of anthraquinone laxatives has been debated. Previously, a few isolated studies have revealed that anthraquinones can cause damage to the structure of colonic epithelial tissue, and long-term intake of anthraquinone laxatives increases colorectal cancer risks. In this study, we focused on sennoside $A$, a primary purgative component in anthraquinone laxatives, and investigated the effects of sennoside $A$ on intestinal inflammation and host metabolism, and we assessed whether it could exacerbate colitis-associated colon cancer in the AOM/DSS mouse model.

Results: Initially, sennoside A disrupted the mucus layer and mechanical barrier of the colon. The dynamic effects of sennoside $A$ on the community structure of gut microbiota were further analysed. We found that sennoside A significantly promoted the dominant growth of Akkermansia muciniphila after 56 days of continuous treatment, which was independent of the prototype of sennoside A and its metabolites, including the main host metabolite rhein and bacterial metabolites. Interestingly, sennoside A suppressed the growth of butyrate-producing bacteria, thereby decreasing butyrate levels. In addition, sennoside A directly inhibited the growth of Clostridium tyrobutyricum and Clostridium butyricum, which was not caused by the prototype of sennoside $A$ but by the direct bacteriostatic effect produced from its metabolite rhein. Last, supplementation with butyrate prevented sennoside A-induced gut dysbiosis and mucus barrier impairment.

Conclusions: Our work reveals that sennoside A can impair the integrity of the intestinal mucosal barrier, which is closely related to the disruption of the structural balance between mucus-degrading bacteria and exogenous fibro-degrading bacteria; this impaired imbalance can induce long-term low-grade inflammation and metabolic disorders associated with tumorigenesis, and it can consequently promote the progression of colonic carcinogenesis in the AOM/DSS mouse model.

\section{Introduction}

Anthraquinone laxatives (e.g., senna products) are commonly employed to relieve chronic constipation and obesity in clinical practice and exist in a variety of herbal/health supplements[1,2]. However, the safety of anthraquinone laxatives has been controversial. As early as 1984, Dufour P et al. pointed out that long-term (16 weeks) administration of sennoside $A$, a primary component of anthraquinone laxatives, could cause damage to the intestinal mucosa, destroy the structure of the intestinal wall, and increase the risk of carcinogenesis in experimental mice[3]. Mori et al. also found that the 1,8dihydroxyanthraquinone contained in rhubarb induced adenomatous hyperproliferation in the colonic and caecal epithelial layers[4]. A prospective study involving 1095 patients found that long-term use of anthraquinone laxatives was associated with a 3.04-fold increased risk of colorectal cancer, which was significantly higher than that of people who did not take this class of drugs[5]; these findings also clearly indicated that herb senna that contains anthraquinone has a considerable colon carcinogenic effect[6]. In addition, in 2001, the National Institutes of Health (U.S.) warned of the potential carcinogenic risk of 
long-term use of anthraquinone compounds[7]. However, there is a lack of clear evidence regarding whether anthraquinone laxatives contribute to the development of tumours.

Of note, intestinal microorganisms play an important role in colonic inflammation and the development of colon cancer, and intestinal mucosal barrier injury is a key initiator of intestinal microorganisms that promote the development of colon cancer $[8,9]$. Once the integrity of the intestinal mucosal barrier is damaged, the bacteria and their metabolites in the intestinal lumen translocate to the intestinal lamina propria through the space between the epithelial cells and trigger the adaptive immune response. During the process, epithelial cells are stimulated to release cytokines such as IL-1, IL- 6 and IL-23. IL-23 further activates Th17 cells. Th17 cells release IL-17 and activate the STAT3 and NF-KB signalling pathways, which promote epithelial cell proliferation and invasion and induce gene mutations, resulting in further destruction of mucosal integrity and an aggravated inflammatory response[10-13]. Chronic inflammation of the colon is a critical step in promoting the advancement of colon cancer. Colon cancer development is the result of a combination of intestinal mucosal barrier damage and an imbalance in intestinal microbial homeostasis[12,13]. Goblet cells formed by the differentiation of colonic epithelial cells continuously secrete and replenish to maintain the coverage of intestinal epithelial cells by the mucus layer to form the first line of defence and lubricate the intestine[8]. The outer layer of mucus is the habitat of commensal microorganisms and the nutrient source of some microorganisms, and the microbial species and their physiological functions in this area can affect the microbial composition in the intestinal lumen[14].

Sennoside $A$ is a representative of anthraquinone laxatives, and gut microbes might play a significant role in the purgative mechanism of sennoside $A$, as sennoside $A$ needs to be hydrolysed in the presence of $\beta$-glucosidase, which can be secreted by gut microbes[2,15]. We hypothesized that sennoside $A$ could interact with the intraluminal microbiota and potentially elevate the pro-inflammatory carcinogenic risk in vivo. In this study, we focused on the role of gut microbes in the integrity of the intestinal mucosal barrier to elucidate whether sennoside A could disrupt the integrity of the colonic epithelial barrier and promote the development of colitis and colon cancer.

\section{Result}

\section{Result 1 Sennoside A induces intestinal epithelial barrier damage to promote bacterial translocation}

To determine whether sennoside A could directly induce colon cancer development, we first evaluated whether long-term (84 days) administration of sennoside A had pathological effects on colon tissue in vivo. The cross section of normal colonic mucosal crypts had a daisy-like morphology [16], and they were distributed with goblet cells that were filled with large amounts of mucus (Figure 1A, as marked by an asterisk). Sennoside A ( $25 \mathrm{mg} / \mathrm{kg}$ ) inhibited the filling of goblet cells with mucus and the infiltration of lymphocytes in the crypt. At a dose of $\sim 50 \mathrm{mg} / \mathrm{kg}$, the colonic mucosa revealed relaxed crypt arrangement, a lack of mucus, and increased lymphocyte crypt invasion. Sennoside A (100 mg/kg) significantly reduced the total number of crypts and increased the distance between the crypt base and mucosal muscles. Extensive infiltration of inflammatory cells (mainly granulocyte neutrophils) was 
observed in the colonic crypt and submucosa in mice treated with $100 \mathrm{mg} / \mathrm{kg}$ sennoside A (Figure 1A\&B). However, colon adenomas were not observed after continuous administration of various doses of sennoside A (25, 50, and $100 \mathrm{mg} / \mathrm{kg}$ ) for 84 days(Figure S1A). Moreover, sennoside A hardly induced a large amount of lymphocyte infiltration in the colonic mucosal layer and heterotype (atypical) hyperplasia (Figure S1B). Thus, sennoside A can lead to changes in the structure and morphology of colonic crypts without active inflammation and the formation of visible adenoma in the colon.

The effect of sennoside A on the mechanical barrier of the colon was further evaluated. As shown in Figure 1C\&D, sennoside A inhibited the expression of claudin-1, ZO-1, and E-cadherin in colonic epithelial tissues in a dose-dependent fashion. To determine whether sennoside A impaired tight junction proteins in colonic epithelial cells, NCM-460 colonocytes were incubated with sennoside in vitro. Sennoside A alone or in combination with $\beta$-glucosidase had no direct effect on tight junctions between NCM-460 cells (Figure S2). The mucus layer is a dynamic barrier that is constantly replenished by the secretory activity of goblet cells. Our analysis was restricted to the inner mucus layer, which is well preserved and thus more reliable for analysis, while the outer layer is prone to be disordered from the diet composition and fixation procedure [17]. The thickness of the inner mucus layer and the number of goblet cells were further evaluated (Figure 1E), mucus thickness could be significantly reduced by sennoside $A$, and the number of goblet cells/crypt was decreased.

Intestinal mucosal permeability changes accurately reflect the degree of damage to the intestinal mucosal barrier and is an important indicator for monitoring the integrity of colonic barrier function[18]. Subsequently, we applied FITC-labelled dextran (FD4) to measure the total intestinal permeability using in vivo imaging technology. We found that sennoside $A$ treatment resulted in the disruption of intestinal barrier function integrity. Compared with the control mice, sennoside A (100 mg/kg) diminished the fluorescence intensity in the intestinal lumen, and the fluorescence showed a diffuse distribution in the abdominal cavity (shown in Figure 1F). Further fluorescence imaging of the colonic epithelium revealed that sennoside A promoted FD4 leakage into colonic epithelial tissue (Figure 1F), accompanied by an increased content of FD4 in serum (Figure 1G). The loss of tight junctions between some colonic epithelial cells further promoted bacterial translocation to the colonic epithelial layer and even invaded into the colonic recess [19]. As shown in Figure $1 \mathrm{H} \& \mathrm{l}$, sennoside A (100 mg/kg) promotes bacterial translocation from the intestinal lumen to the colonic epithelial layer. Taken together, our data indicate that sennoside A destroys the functional barrier of the colonic mucosa, increases the permeability of the colonic barrier and promotes the translocation of microorganisms in the intestinal lumen.

\section{Result 2 Sennoside A promotes the dominant growth of Akkermansia muciniphila}

It has been pointed out that the gut microbiota involved in the integrity of the intestinal mucosal barrier is the initial link to the occurrence and development of colitis-associated colon cancer [13]. We performed 16S rDNA detection analysis of bacteria from mouse caecal contents. As shown in Figure S3A\&B, sennoside $A(100 \mathrm{mg} / \mathrm{kg}$ ) treatment for 84 days changed the microbial community structure and decreased microbial alpha diversity in mice. Further analysis was performed to obtain the bacterial 
characteristics of the pathological phenotype generated by sennoside A. As shown in Figure 2A, sennoside A significantly promoted the relative abundance of Verrucomicrobia and reduced the relative abundance of Firmicutes, while Akkermansia was the only genus of Verrucomicrobia among colonic microorganisms. The results of the genus-level analysis indicated that the relative abundance of the Akkermansia genus was significantly promoted by sennoside A (Figure 2B and Figure S3C).

In addition, linear discriminant analysis effect size (LEfSe) was used to determine that the representative taxon of the sennoside A group was Akkermansia muciniphila under the Verrucomicrobia phylum, and the evolutionary clade diagram displayed consistent results (Figure 2C). Based on quantitative PCR, we observed that the absolute $A$. muciniphila count significantly increased following sennoside A treatment (Figure 2D). To further explore the effects of sennoside $A$ on the dynamic growth of A. muciniphila, 16S rDNA sequencing was performed at different time points. The results showed that successive administration of sennoside $\mathrm{A}(100 \mathrm{mg} / \mathrm{kg})$ for 56 days caused a dominant growth of $A$. muciniphila (Figure 2E). However, the dominant growth of $A$. muciniphila did not occur immediately after sennoside $A$ treatment. Presumably, sennoside A might not directly affect the growth of $A$. muciniphila.

To investigate whether sennoside $A$ had a direct impact on the growth of $A$. muciniphila, bacterial growth was evaluated at OD600[20] (Figure 2F). Sennoside A is metabolized by a variety of bacteria-driven hydrolytic enzymes in the intestinal lumen, so the SenA-CM group was used to simulate the sennoside A metabolic mixture in vitro (Experiment 1). To rule out the effect of normal faecal supernatant on the growth of $A$. muciniphila, the faecal supernatant was set up as a control group (Experiment 2). Rhein, an important end product of sennoside A subjected to $\beta$-glucosidase hydrolysis, was simultaneously evaluated (Experiment 3) [15]. There was no significant difference between the Sen-CM and Ctrl-CM groups in the growth of $A$. muciniphila at different time points (Figure 2F). Sennoside $\mathrm{A}$ and rhein both had no effect on the growth of $A$. muciniphila at different time points. Taken together, the dominant growth of $A$. muciniphila was not directly induced by sennoside $\mathrm{A}$ and its metabolites.

\section{Result 3 Sennoside A-mediated decrement of Clostridiales causes butyrate reduction.}

Further genus-level STAMP difference analysis (Welch's $t$-test, $P<0.05$ ) was performed [21]. The genuses that were increased in number following sennoside A treatment (Akkermansia and Sutterella) were consistent with biomarkers derived from LEfSe analysis, whereas the suppressed taxons Coprococcus, Prevotella, Oscillospira, and Ruminococcus are mainly involved in dietary fibre metabolism to synthesize SCFAs (Figure S4 and Table S1). An order level analysis revealed that sennoside A significantly inhibited the relative abundance of Clostridiales (Figure $3 \mathrm{~A}$ ), which is the most predominant butyrate-producing taxon. Considering that the dominant growth of $A$. muciniphila was identified on the 56th day of continuous sennoside A treatment and sennoside A had no direct effect on A. muciniphila in vitro, we further observed the dynamic effects of sennoside A on Clostridiales. As shown in Figure 3B, the significant inhibitory effect of sennoside A on Clostridiales growth occurred at 28 days, which is earlier than when A. muciniphila dominant growth occurs. Based on this speculation, sennoside A was superior 
at promoting A. muciniphila growth, which was a secondary effect of its growth inhibition on Clostridiales.

To determine whether sennoside $\mathrm{A}$ has a direct inhibitory effect on Clostridiales growth, we further performed an in vitro growth assay by selecting two major butyrate producing species: Clostridium tyrobutyricum and Clostridium butyricum. As shown in Figure 3D\&E, the sennoside A prototype had little effect on the growth of $C$. butyricum and $C$. tyrobutyricum, and there was no significant difference. SenA$\mathrm{CM}$ began to significantly inhibit growth of $C$. butyricum at a concentration of $6.25 \% 24$ hours after administration of sennoside A metabolites (24 h; 6.25\% SenA-CM vs 6.25\% Ctrl-CM, ${ }^{*} P<0.05$ ), and at $50 \%$ concentration, it inhibited the growth of $C$. tyrobutyricum ( $24 \mathrm{~h} ; 50 \%$ SenA-CM vs $50 \%$ Ctrl-CM, $* \star P<0.01$ ). In addition, rhein, the major metabolite of sennoside $A$, significantly inhibited the growth of $C$. butyricum at $0.5 \mu \mathrm{M}$ at 24 hours $(24 \mathrm{~h} ; 0.5 \mu \mathrm{M} v s 0 \mu \mathrm{M}, \# \# P<0.01)$, and there was significant inhibition of $C$. tyrobutyricum at $2 \mu \mathrm{M}(24 \mathrm{~h} ; 2 \mu \mathrm{M} v s 0 \mu \mathrm{M}, \# P<0.01)$. After 48 hours of exposure, the growth inhibition efficacy of SenA-CM with rhein on $C$. butyricum and $C$. tyrobutyricum decreased. SenA-CM required up to $50 \%$ concentration to produce significant inhibition of $C$. butyricum and $C$. tyrobutyricum. The potency of rhein on $C$. butyricum and $C$. tyrobutyricum has also increased. In this study, we found that sennoside $A$ can directly inhibit clostridium in the intestine, which was not mediated by its prototype but by the primary metabolite rhein.

Next, short-chain fatty acid levels of SCFAs were quantified to verify that microbial fibre metabolism was disrupted by sennoside A. We observed significantly lower concentrations of the SCFAs butyric acid, valeric acid and isovaleric acid in mice treated with sennoside A (Figure 3D and Figure S5), which were consistent with the decreased abundance of butyric-producing bacteria. Overall, sennoside A may decrease the abundance of butyric-producing bacteria by affecting intestinal bacterial community structure, thus reducing the content of intestinal butyric acid.

\section{Result 4 Sennoside A-induced intestinal microbial disturbance is a key step in intestinal mucosal barrier function damage}

To elucidate whether sennoside A-generated damage to the colonic mucus barrier is dependent on intestinal bacterial homeostasis, we performed a faecal bacteria transplantation experiment. After initial antibiotic cocktail treatment, mice received a weekly microbial transplant from the sennoside $A$ group (SenA-ABX) and control group (Ctrl-ABX) (Figure 4A). We observed that colonic mucus layer thickness and goblet cell number were significantly inhibited in SenA-ABX mice in comparison with Ctrl-ABX mice (Figure 4B). Live imaging of FD4-gavaged mice revealed that faecal bacteria derived from the sennoside A group resulted in the disruption of intestinal barrier integrity with a diffused distribution in the abdominal cavity. Moreover, FD4 leaked into colonic epithelial tissue, and the FD4 content in the serum increased (Figure 4C\&D). In addition, faecal bacteria transplantation after sennoside A (100 mg/kg) treatment significantly promoted the translocation of intraluminal microorganisms into colonic epithelial tissue (Figure 4E\&F). 
To determine whether short-chain fatty acid content was closely related to the alterations in intestinal bacteria affected by sennoside $A$, we further examined the potential reduction in the content of multiple short-chain fatty acids. The results were consistent with those of animals chronically treated with sennoside $A$; faecal bacteria transplantation derived from the sennoside $A$ group did not affect changes in acetic acid content but decreased valeric and butyric acid content compared with that of the Ctrl and Ctrl-ABX groups (Figure 4G). The results showed that the change in short-chain fatty acid content, such as butyric acid, by sennoside A was closely related to its effect on intestinal bacteria. Further analysis of the bacterial community structure, as shown in Figure S6A-E, showed that the sennoside A $(100 \mathrm{mg} / \mathrm{kg})$ faecal bacteria transplantation group (SenA-ABX) and the sennoside $A(100 \mathrm{mg} / \mathrm{kg}$, SenA) group had lower a-diversity than the control group (Figure S6F). The results of $\beta$-diversity analysis (PcoA, Weighted Unidrac) showed that the composition of the SenA-ABX bacteria was similar to that of the SenA group over a long duration and that it significantly grouped with that of Control group bacteria (Figure $4 \mathrm{H}$ ).

\section{Result 5 Butyrate partially shapes gut dysbiosis and ameliorates intestinal epithelial barrier impairment in sennoside A-treated mice}

The short-chain fatty acids butyric acid, acetic acid and propionic acid are the major end products of dietary fibre, which provide approximately $10 \%$ of the calories we absorb [22]. Mucin 0-glycans serve as an endogenous fermentation substrate for butyrate production [23]. Based on the aforementioned results, we hypothesized that the lower butyrate levels triggered by the decrease in butyrate-producing bacteria may be the reason for the increase in A. muciniphila. Since the abundance of Clostridiales was dramatically reduced after 28 and 42 days of sennoside A treatment, we treated mice with sennoside $A$ for 4 weeks and supplemented their drinking water with $150 \mathrm{mM}$ sodium butyrate (Bt-SenA) [24,25] (Figure 5A). Consequently, the microbial community structures in sennoside A-treated mice with sodium butyrate were different from those in sennoside A mice, but the microbial alpha diversity was not affected (Figure S7A\&B). Clostridiales was the hallmark bacterium of the Bt-SenA group rather than A. muciniphila (Figure 5B and Figure S7C), and the relative abundance of Clostridiales was significantly increased. (Figure S7D).

We next tested whether oral administration of sodium butyrate prevented sennoside A-induced intestinal epithelial barrier impairment in C57BL/6 mice. In line with our expectations, the mucosal barrier damage phenotype in the mucous layer, permeability, bacterial translocation and mechanical barrier were alleviated in sennoside A-treated mice (Figure 5C-G and Figure S8).

\section{Result 6 Sennoside A induces low-grade inflammation as well as metabolic disorders}

When the intestinal barrier is damaged, intestinal barrier permeability increases and mucus secretion decreases; then, bacteria and their secreted metabolites have a higher probability of directly contacting epithelial cells, invading the mucosal layer, and activating the body's immune response [26,27]. After sennoside A treatment, body weight and the epididymis adipose mass were significantly reduced, which might be related to its purgative and weight-loss efficacy (Figure 6A and Figure S9A). In addition, we found that sennoside $A$ was able to significantly reduce colon length, which was nearly comparable to the 
colon length of mice in the DSS chronic colon inflammation model group (Figure 6B). To determine whether sennoside A could induce low-level inflammation, we examined faecal lipocalin (LCN2), a neutrophil protein that binds bacterial siderophores and is a sensitive and broadly dynamic marker of low-grade inflammation in mice [28]. Faecal LCN2 level was increased in the group treated with $50 \mathrm{mg} / \mathrm{kg}$ and $100 \mathrm{mg} / \mathrm{kg}$ sennoside $A$ at 56 days. On day $84,100 \mathrm{mg} / \mathrm{kg}$ sennoside A caused a dramatic increase in LCN2 (approximately 10- to 15-fold vs Ctrl) (Figure 6C.). By using a Luminex assay to detect ten inflammatory factors in mouse serum, we found that sennoside $A$ was able to induce an increased level of a variety of proinflammatory factors, including IFN-y, MIP-2, IL-17 and GM-CSF (Figure 6D). Further examination of the number of Th1, Th2, and Th17 cells in mesenteric lymph nodes revealed that sennoside A resulted in a significant increase in the proportion of Th1 cells as well as Th17 cells but not Th2 cells (Figure 6E\&F and Figure S9C). The increase in Th17 cells is also consistent with the decline in butyrate due to the ability of butyrate to activate GPR109a, thereby inhibiting Th17 cells [29]. Although sennoside $A$ does not cause active colitis, low-grade intestinal inflammation induced by sennoside $A$ is associated with an increased incidence of colon cancer[28,30]. The proliferation of epithelial cells, measured by Ki67 expression, was significantly increased in sennoside A-treated mice, and it was ameliorated by sodium butyrate (Figure S10). The above results demonstrate that long-term sennoside $A$ treatment induces chronic low-grade inflammation.

Metabolic disorders are associated with and defined by elevated chronic low-grade inflammation, which can also affect the incidence of colon cancer [31,32]. To assess metabolic alterations in response to microbial dysbiosis and low-grade inflammation induced by sennoside A, untargeted metabolome profiles were generated from faecal samples. Distinct clustering of metabolites was apparent between the control and sennoside A-treated mouse groups in a principal component analysis model, with distinct separation of the two groups (Figure 7A). Sennoside A treatment was sufficient to trigger widespread changes in metabolites, and 50 differential metabolites were obtained based on a fold change greater than 2 and a $P$ value less than 0.05 (Figure S11 and supplementary dataset S1). The topology map generated by MetaboAnalyst (www.metaboanalyst.ca) described the impact of sennoside A on these responsive metabolites and revealed the most significant pathways, including purine metabolism, pantothenate and CoA biosynthesis, beta-alanine metabolism, arginine biosynthesis, and butanoate metabolism (Figure 7B). The levels of thymine, xanthosine, creatinine, and orotic acid were significantly elevated in the faeces of the sennoside A groups compared to the control mice, and these factors are key nodes of the altered metabolic pathway (Figure 7C).

\section{Result 7 Sennoside A exacerbates carcinogenesis in a colitis-associated cancer model}

Using the azoxymethane (AOM)/DSS chemical-induced colon cancer model, we evaluated the effect of sennoside $A$ on the promotion of colon cancer. As shown in Figure 8A\&B, colon length and body weight were significantly reduced in the AOM/DSS and AOM/DSS/sennoside A groups compared with the control group, while there was no significant difference between the two groups. Notably, colonic adenomas were already evident in the AOM/DSS/sennoside A group at 40 days of treatment, while only a few adenomas were produced in the animals in the AOM/DSS group. In addition, the tumour volume in 
the sennoside A-treated group was significantly increased compared with that in the AOM/DSS group, indicating that sennoside $A$ already had a certain promoting effect on the occurrence of colon tumours at 40 days (Figure 8C\&D). After sacrificing the animals at day 90, we found that the number and size of colonic adenomas were significantly increased in the presence of sennoside A. Although adenomas had also occurred in the AOM/DSS group, sennoside A significantly promoted the development and progression of colonic tumours. Furthermore, we found a dramatic increase in LCN2 in faeces in the AOM/DSS and AOM/DSS/sennoside A groups at day 40. The AOM/DSS/sennoside A group had a tendency to increase LCN2 levels compared with those of the AOM/DSS group at the same time point, with an increase in the number of tumours; these results suggest that the low level of inflammation caused by sennoside A may promote tumour formation. The LCN2 content tended to be similar in both groups at 90 days, which was possibly due to increased inflammation caused by long-term DSS consumption; thus, the low-level inflammatory effect caused by sennoside A was weakened and could not be reflected as an accurate indicator (Figure 8E\&F). Increased Th17 cell infiltration in the mesenteric lymph nodes and increased IL-17 secretion in the serum suggest that sennoside A may increase the phosphorylation level of STAT3 by activating the JAK2/STAT3 signalling pathway, thereby activating the transcription of downstream genes related to cell survival, such as BCL2, BAX, and BCL2L1, conferring cells in an overactive proliferative state[24,25]. As shown in Figure $\mathbf{8 G}$, we found that the AOM/DSS/sennoside A group had significantly higher levels of p-Stat3 than the AOM/DSS group. In addition, Wnt/ $\beta$-catenin plays an important role in cell proliferation as well as tumorigenesis, and downstream target genes of $\beta$-catenin, such as $M y c$ and $C c n d 1$, can also promote the level of cell proliferation [33,34]. Figure $\mathbf{8 G}$ also shows that sennoside A remarkably upregulated the expression level of $\beta$-catenin. By examining the proliferation levels of tissues adjacent to colon tumours, we found that sennoside $A$ resulted in an increase in the level of epithelial cell proliferation near the tumour tissue, suggesting a possible malignant change in proliferation of epithelial cells (Figure $\mathbf{8 H}$ ). Collectively, our data demonstrate that sennoside $A$ has a tumour-promoting effect in colitis-associated colonic carcinoma.

\section{Discussion}

Anthraquinone laxatives (i.e., senna) and purgative botanicals (rhubarb, aloe vera, and senna) that contain anthraquinone compounds are used frequently in the clinic, but the possible risk of anthraquinone use for the development of colorectal neoplasms has been controversial for a long time $[3,4,7]$. Previous studies with infrequent experimental data indicate that anthraquinone causes damage to the structure of the colonic epithelial tissue. Prospective study data also indicate that long-term anthraquinone use has a certain correlation with colon cancer development. However, limited evidence cannot positively interpret the potential adverse effects of anthraquinone laxatives. In this study, we innovatively paid attention to the integrity of the intestinal mucosal barrier, the first line of defence against commensal microorganisms and invading pathogens. We observed that sennoside A can disrupt the intestinal mucosal barrier by disrupting the homeostasis of intestinal bacteria and cause the production of intestinal epithelial inflammation, thereby promoting the development of colon cancer. 
Starting from the key initial link to the occurrence of colon cancer, microbiota-mediated mechanisms that connect long-term intake of anthraquinone laxatives to the progress of gastrointestinal inflammation and cancer have not been described.

The loss of integrity of the intestinal mucosal barrier is a key factor in the development of colon cancer in intestinal microbiota-mediated colitis. The intestinal mucosal barrier is usually divided into four barrier systems: the mechanical barrier, chemical barrier, microbial barrier and immune barrier [35]. All colonic pathologies arise from damage to the physical barrier (including the mechanical barrier and the mucus layer in the chemical barrier), of which the mucus layer is the first line of defence to insulate the intraluminal microbiota against direct contact with colonic epithelial tissue. Microorganisms are in direct contact with the colonic epithelial tissue when the first line of defence is breached, promoting an increase in colonic permeability and bacterial translocation to the epithelial layer and further inducing colonic inflammation and metabolic disorders. Long-term low-grade recurrent inflammation can activate cancerpromoting signalling axes such as STAT3 and $\beta$-Catenin, further promoting the development of colon cancer[36,37].

Here, we have shown that long-term (84 days) administration of sennoside A (50 and $100 \mathrm{mg} / \mathrm{kg}$ ) can promote changes in the structure and morphology of colonic crypts and promote the expression and distribution of tight junction protein (ZO-1, claudin-1) and adhesion protein (E-cadherin) in colonic epithelial tissues but does not directly induce damage to colonic epithelial cells. Consistently, sennoside A induces damage to the physical barrier of the colon, disrupts colonic barrier function and promotes increased colonic permeability as well as bacterial translocation to the colonic epithelium.

Given that the role of gut microbes in the colonic mucosal barrier cannot be ignored, we examined the effect of sennoside A on the gut microbial community structure. Sennoside A significantly promoted the dominant abundance of Verrucomicrobia phyla and significantly inhibited the relative abundance of Firmicutes. Lefse analysis showed that A. muciniphila is the hallmark biomarker of the sennoside (100 $\mathrm{mg} / \mathrm{kg}$ ) group. A. muciniphila is a key species of the mucosymbiotic microbiome, a class of exclusive mucin-degrading bacteria that depends on mucins as a sole carbon source. Therefore, it may be reasonable that damage to the integrity of the mucus barrier "wall" and the "channel" can enable microbial translocation to epithelial tissues. Sennoside A promotes the dominant growth of Akkermansia muciniphila, which disrupts the dynamic balance of colonic mucus synthesis and degradation. Considering the sensitivity of dynamic alterations in the gut microbiome composition, faecal microbial community dynamics show that it is an incremental process of dominant growth in A. muciniphila by sennoside A. At the early stage of sennoside $A$ administration, there were no significant effects in the relative abundance of $A$. muciniphila, but a significant growth-promoting effect began to appear after 56 days of continuous gavage. Does sennoside A directly promote the growth of $A$. muciniphila? Further in vitro flora culture confirmed that the prototype of sennoside $A$ and the metabolic mixture of sennoside $A$ and the main metabolite rhein had no significant effect on the growth of $A$. muciniphila. 
In addition, the sennoside A-medited growth inhibition of several SCFA-producing genera, especially butyrate-producing bacteria, occurs prior to the promotion of $A$. muciniphila growth. Butyrate is the preferred energy source for colonocytes and is locally consumed [37]. Acetate production by Akkermansia muciniphila also serves as an energy substrate [38]. Presumably, the promotion of sennoside $\mathrm{A}$ on $A$. muciniphila is a secondary reaction to its inhibition of Clostridiales growth because of the change in energy source for colonocytes in the absence of butyric acid. Further investigation of the direct bacteriostatic effect of sennoside $A$ in vitro by two major butyric acid-producing bacteria (Clostridium tyrobutyricum and Clostridium butyricum) showed that sennoside $A$ in the intestine could directly inhibit the growth of $C$. tyrobutyricum and $C$. butyricum not by unchanged sennoside $A$ but by a direct bacteriostatic effect produced by its metabolites, especially rhein. Consistent with the microbiota analysis data, the decrease in the abundance of butyrogenic bacteria resulted in a decrease in butyric acid levels. Butyrate absence also leads to impairment of intestinal barrier function. To clarify whether the disturbance of the microbiota caused by sennoside $A$ is the key to the degradation of mucin and the disruption of the intestinal mucosal barrier, the study evaluated the contribution of intestinal microorganisms in this process by faecal microbiota transplantation (FMT). Furthermore, butyrate exerts protective effects on intestinal homeostasis and intestinal barrier function. These findings provide strong evidence that intestinal microorganisms and their metabolites of sennoside A are involved in destabilized intestinal mucosal barrier integrity.

Since sennoside A induces the advancement of low-grade inflammation in the colon, we next tested whether there was a link between butyrate loss and inflammation. To this end, the secretion of various inflammatory factors in the serum of mice was detected using a liquid chip, and we found that sennoside A could induce an increase in the levels of various proinflammatory factors, such as the low-level inflammatory markers LCN2 and IL-17, IFN- $\gamma$, and MIP-2, and an increase in the number of Th1 and Th17 cells, further indicating the low-level inflammation caused by sennoside A. The present work highlights that low-grade inflammation can also agitate metabolic disorders in the body and contribute to the development of a variety of diseases $[39,40]$. Based on metabolomics studies, sennoside $A$ is found to cause metabolic abnormalities related to purine metabolism, pyrimidine metabolism and a variety of amino acid metabolism. Our results suggest that a long-term state of low-level inflammation is responsible for the probability of colon tumours. Taken together, we speculated that sennoside A may not lead to tumorigenesis in a delayed manner, and its effect on the low level of inflammation caused by intestinal bacteria may be able to promote the development of colon cancer.

Sennoside A alone did not induce colonic inflammation or adenoma development. However, we observed typical changes featured by the chronic low-level inflammation state induced by sennoside $A$, which might accelerate the development of colon tumours [1]. Further studies with the AOM/DSS chemicalinduced carcinogenesis model found that sennoside A promoted the occurrence and development of colon cancer. AOM/DSS mice treated with sennoside A developed tumours earlier than control mice, and the AOM/DSS model applied to C57BL/ 6 mice usually required three rounds (approximately 60 days) of DSS induction to form colonic adenomas. Significantly, sennoside A shortened the process. At the end of the experiment (90 days), we also found an increase in tumour number and size that was accompanied 
by a more intense activation of STAT3 and $\beta$-catenin signalling pathways, and promoted the proliferation of epithelial cells near the tumour, suggesting a possible malignant transition. At 40 days, tumour occurrence and volume were correspondingly increased, indicating that the low-grade inflammatory process in the colon was activated by sennoside $A$, which was consistent with previous studies on the carcinogenic effect of sennoside A.

\section{Conclusion}

In summary, our results provide evidence for the inflammatory carcinogenic risk of sennoside $A$, a representative component of anthraquinone laxatives. Sennoside A induced a decrease in butyricproducing bacteria and consequently increased the growth of $A$. muciniphila, which disrupted intestinal mucosal barrier function, initiated low-grade inflammation and metabolic disorders, and ultimately promoted the development of colon tumours (Figure 9). Our study emphasizes that sennoside A promotes the progression of colon cancer by disrupting the balance between mucus-degrading bacteria and butyrate-producing bacteria and impairing intestinal mucosal barrier integrity to induce colonic inflammation production.

\section{Materials And Methods}

\section{Drug administration and animal experiments}

The sennoside A (CAS. 81-27-6; HPLC 98.58\%; stored at 2-8 ${ }^{\circ} \mathrm{C}$ ) used in this study was purchased from Biopurify Phytochemicals Ltd., China.

Animal experiments were conducted in accordance with the Institutional Animal Care and Use Committees of Nanjing University of Chinese Medicine and followed guidelines issued by the National Institutes of Health. Six-week-old C57BL/6J male mice (18 to $20 \mathrm{~g}$ ) were purchased from the Model Animal Research Center of Nanjing University (Nanjing, China). Mice were housed under standard laboratory conditions (room temperature: $22 \pm 2^{\circ} \mathrm{C}$; humidity: $50 \pm 5 \%$ ) with a light/dark cycle of $12 / 12 \mathrm{~h}$. Before the experiment, they were acclimatized to the environment and had free access to food and water for at least 3 days.

\section{Animal protocol 1: Effects of sennoside A on direct effects in mice}

Mice were randomly distributed and were administered sennoside A daily at $0,25,50$, or $100 \mathrm{mg} / \mathrm{kg}$ by intragastric gavage for 12 weeks. Faecal samples from each group were collected weekly and stored at $-80^{\circ} \mathrm{C}$. Microbial abundances were averaged across time points as shown in Figures $2 \& 3$. At the end of the experiment, the mice were sacrificed, and blood and colon tissues were collected for analysis.

\section{Animal protocol 2: Antibiotic treatment and faecal microbiota transplantation}


For in vivo antibiotic pretreatment, six-week-old male C57BL/ $6 \mathrm{~J}$ mice were supplemented with the antibiotics vancomycin ( $0.5 \mathrm{~g} /$ /itre), neomycin sulfate $(1 \mathrm{~g} /$ /itre), metronidazole ( $1 \mathrm{~g} /$ litre), and ampicillin $(1 \mathrm{~g} /$ litre), which was provided in drinking water for five days.

After antibiotic treatment, faecal transplant was performed on the basis of an established protocol [41]. Briefly, 6-week-old male donor mice were given NS and sennoside A (100 mg/kg) for 12 weeks. During weeks 10 to 12 (total of 14 days), faecal microbiota from each donor mouse was collected daily and stored at $-80^{\circ} \mathrm{C}$. The stools from donor mice of each group were pooled, and $150-180 \mathrm{mg}$ was resuspended in $1 \mathrm{ml}$ of sterile saline. The solution was vigorously mixed for $10 \mathrm{~s}$ using a benchtop vortex (MS 3, IKA, Germany) and then was centrifuged at $800 \mathrm{~g}$ for $3 \mathrm{~min}$. The supernatant was collected and used as transplant material as described below. Fresh transplant material was prepared on the same day of transplantation within 10 min before oral gavage to prevent changes in bacterial composition. Before being killed for subsequent analysis, six-week-old male recipient mice were inoculated weekly with fresh transplant material (100 $\mu$ l for each mouse) by oral gavage for 12 weeks. (Figure 4)

\section{Animal protocol 3: Butyrate supplement in sennoside A-treated mice.}

Mice were maintained with sennoside A-treatment for four weeks and divided into two subgroups (10 mice/group). The two groups continued to be treated with sennoside $A$ and simultaneously were given $150 \mathrm{mM}$ sodium butyrate or placebo to drink for the last eight weeks according to our microbiota analysis of compositional dynamics.

\section{Animal protocol 4: The effect of sennoside A on AOM/DSS-induced colon cancer in mice.}

C57BL/ 6 male mice (age, 6 weeks) were treated daily with sennoside A (100 mg/kg) or saline by oral gavage throughout the experiment. After 1 week of intraperitoneal injection with AOM $(10 \mathrm{mg} / \mathrm{kg}$; SigmaAldrich), the mice were given $1.5 \%$ DSS in drinking water for 5 days, which was followed by access to regular drinking water for 10 days. This cycle was repeated three times, and the mice were sacrificed for analysis of colon tumorigenesis at days 40 and 90 after AOM injection.

\section{Haematoxylin and eosin staining and histopathologic analysis}

Fresh intestinal mouse tissue was fixed in Carnoy's fixative solution (60\% methanol, $30 \%$ chloroform, $10 \%$ glacial acetic acid) for at least 24 hours and then was processed into paraffin-embedded tissue sections. After Carnoy's fixation, tissues were sectioned at a thickness of $5 \mu \mathrm{m}$ using standard protocols. To determine the extent of tissue damage, H\&E-stained slides were scored on a scale of 0-3 based on four parameters: $3=$ markedly increased, $2=$ moderately increased, $1=$ slightly increased and $0=$ normal. The scoring parameters indicated the amount of crypt atrophy (including the number and depth of crypt), the amount of polymorphonuclear leukocyte infiltrate in crypt, the counts of goblet cells per crypt, and the lymphocytes at the basement of crypt and the mucous membrane base.

\section{The colonic mucus layer measurements}


To measure the thickness of the colonic inner mucus layer, colonic tissues were sectioned at a thickness of $5 \mu \mathrm{m}$ and then were deparaffinized. The sections were stained using an Alcian blue staining kit (Leagene, Beijing, China), and anti-Muc2 (sc-515032, Santa Cruz Biotechnology) immunofluorescent staining was performed according to the manufacturer's instructions.

\section{Measurement of intestinal permeability with FITC-Dextran}

Fluorescence detection in living, anesthetized mice was captured with a IVIS ${ }^{\mathrm{TM}}$ liveimaging system (IVIS Lumina III, PerkinElmer). Mice were gavaged with fluorescein-isothiocyanate (FITC)-dextran (4kDa; Sigma) at a doseage of $400 \mathrm{mg} / \mathrm{kg}$ and then animals were studied $1 \mathrm{hr}$ later using multispectral fluorescent capture. Blood samples were obtained after 4-5hr by retro-orbital bleeding, and the fluorescence intensity in the serum was measured at an excitation wavelength of $485 \mathrm{~nm}$ and an emission wavelength of $520 \mathrm{~nm}$ using a Microplate System (EnSpire, Perkin Elmer). FITC-dextran diluted in PBS was used to plot a standard curve, and the serum concentration of FITC-dextran was calculated. Furthermore, the mouse ileal-tissue sections were fixed with Carnoy's fluid and thin sections ( $5 \mathrm{~mm})$ were cut and deposited on glass slides. Images were acquired using Inverted fluorescence Microscope (Alexa Fluor 488, Axio vert A1, ZEISS).

\section{Analysis of tight-junction proteins}

The tissue sections and NCM-460 cell were immunostained with specific antibodies (claudin-1 (A-9) (mouse) Santa cruz sc-166338; ZO-1 (D6L1E) Rabbit mAb CST 13663 ; E-Cadherin (4A2) Mouse mAb, CST 14472) by incubating overnight at $4^{\circ} \mathrm{C}$. Following antibody incubation, slides were incubated with Alexa Fluor 594 or 488 -conjugated secondary antibody for $2 \mathrm{hr}$ at room temperature followed by washing three times with PBS (3 cycles, $5 \mathrm{~min}$ ). The nuclei were stained with DAPI and slides were mounted in ProLong antifade reagent. Markings were detected and photographed by Inverted fluorescence Microscope (Axio vert A1, ZEISS).

To extract proteins from ileal epithelial cells, the epithelial cell fraction from ileal tissues were isolated. Total protein lysates were fractionate $\mathrm{d}$ by $10 \%$ SDS-PAGE and electro-blotted onto polyvinylidene difluoride membranes (Immobilon TM-P; Millipore, USA). Afterwards, the membranes were blocked with $5 \%$ non-fat milk for $1 \mathrm{~h}$ at room temperature in TBST buffer $(10 \mathrm{mM}$ Tris, $150 \mathrm{mM} \mathrm{NaCl}, \mathrm{pH} 7.6,0.1 \%$ Tween 20) and probed with primary antibodies overnight at $4^{\circ} \mathrm{C}$. The membranes were then incubated with horseradish peroxidase-conjugated secondary antibody. The dilutions of primary and secondary antibodies have been described in the Antibody subsection above. Protein bands were developed using enhanced chemiluminescence reagent (Millipore). The blots were probed with the primary antibodies against GAPDH (Bioworld, China), claudin-1, ZO-1 and E-Cadherin (instrument same as above).

\section{Bacterial Translocation by Fluorescent in situ hybridization (FISH)}

Mucus immunostaining was paired with fluorescent in situ hybridization (FISH), as previously described [42], in order to analyze bacteria localization at the surface of the intestinal mucosa. Five- $\mu \mathrm{m}$ sections 
were cut and dewaxed by preheating at $60^{\circ} \mathrm{C}$ for $10 \mathrm{~min}$, followed by bathing in xylene at $60^{\circ} \mathrm{C}$ for $10 \mathrm{~min}$, xylene at room temperature for $10 \mathrm{~min}$ and $99.5 \%$ ethanol for $10 \mathrm{~min}$. After deparaniffization and rehydratation, sections were incubated in hybridization buffer $[20 \mathrm{mM}$ Tris- $\mathrm{HCl}, 0.9 \mathrm{M} \mathrm{NaCl}$ and $0.1 \%$ SDS (pH 7.2)] $10 \mathrm{~min}$ at $50^{\circ} \mathrm{C}$. Next, sections were incubated with $100 \mathrm{nM}$ EUB338I probe (Sequence 5'-GCT GCCTCC CGT AGG AGT-3', FITC-conjugated, FB-0010B, EXONBIO) in hybridization buffer in the dark, overnight at $42^{\circ} \mathrm{C}$. After washing for $10 \mathrm{~min}$ in wash buffer $(20 \mathrm{mM}$ Tris- $\mathrm{HCl}, \mathrm{pH} 7.4,0.9 \mathrm{M} \mathrm{NaCl})$ and $3 \times 10$ min in PBS, Mucin 2 primary antibody was diluted to 1:200 in block solution and applied overnight at $4{ }^{\circ} \mathrm{C}$. After washing in PBS, block solution containing anti-mouse Alexa 594 secondary antibody (A21203, Invitrogen) and Hoechst 33258 was applied to the section for $3 \mathrm{~h}$. Observations were performed with a Zeiss-Axio vert A1 microscope. The distance between bacterial populations and the epithelial surface was measured at four points of the proximal, middle and distal colon in each mouse.

\section{Gut Microbiota Analysis by 16 S Sequencing}

Stool samples ( $n=5$ per group) were snap-frozen in liquid nitrogen before storage at $-80^{\circ} \mathrm{C}$. Total genomic DNA was extracted from samples using the CTAB/SDS method. DNA concentration and purity were monitored on a $1 \%$ agarose gel, and then the DNA was diluted to $1 \mathrm{ng} / \mu \mathrm{l}$ using sterile water. $16 \mathrm{~S}$ rRNA genes were amplified using specific primers with barcodes. All PCRs were carried out in a $30 \mu \mathrm{L}$ volume comprising $15 \mu \mathrm{L}$ of Phusion High-Fidelity PCR Master Mix (New England Biolabs), $0.2 \mu \mathrm{M}$ of forward and reverse primers, and approximately $10 \mathrm{ng}$ of template DNA. The thermal cycling consisted of initial denaturation at $95^{\circ} \mathrm{C}$ for $3 \mathrm{~min}$, followed by 25 cycles of denaturation at $95^{\circ} \mathrm{C}$ for $30 \mathrm{~s}$, annealing at $55^{\circ} \mathrm{C}$ for $30 \mathrm{~s}$, elongation at $72^{\circ} \mathrm{C}$ for $30 \mathrm{~s}$, and finally an incubation at $16^{\circ} \mathrm{C}$ for $2 \mathrm{~min}$. The $\mathrm{V} 3$ and $\mathrm{V} 4$ hypervariable regions of the $16 \mathrm{~S}$ RNA gene were amplified. The same volumes of $1 \times$ loading buffer (containing SYBR green) and PCR products were mixed, and electrophoresis was performed on a $2 \%$ agarose gel. Samples with bright signals of $460 \mathrm{bp}$ in length $(\mathrm{V} 3+\mathrm{V} 4)$ were chosen for further experiments. PCR products were mixed in equivalent ratios. Then, the mixture of PCR products was purified with a GeneJET Gel Extraction Kit (Thermo Fisher Scientific, USA). Sequencing libraries were generated using an NEB Next Ultra DNA Library Prep Kit for Illumina (NEB, USA) following the manufacturer's recommendations, and index codes were added. The library quality was assessed on a Qubit@ 2.0 Fluorometer (Life Technologies, CA, USA) and an Agilent Bioanalyzer 2100 system. Finally, the library was sequenced on an Illumina MiSeq platform, and 250 bp paired-end reads were generated.

Quality filtered reads were analysed with the software package QIIME[43]. High-quality reads for bioinformatics analysis were selected, and all of the valid reads from all samples were clustered into operational taxonomic units (OTUs) based on $97 \%$ sequence similarity. a-Diversity was calculated based on the Chao1 diversity index. The variation between the experimental groups ( $\beta$-diversity) was assessed with principal coordinate analysis (PCoA) plots. Bacterial genera with statistically significant differences were assessed using linear discriminant analysis effect size (LefSe) (http://huttenhower.sph.harvard.edu/galaxy).

\section{Bacterial growth assays of strains subjected to different treatments}




\section{Bacterial strains and culture conditions:}

Akkermansia muciniphila, type strain, DMS 22959. The strain was cultured at $37^{\circ} \mathrm{C}, 10 \% \mathrm{H}_{2}, 5 \% \mathrm{CO}_{2}$ and $85 \% \mathrm{~N}_{2}$, with medium $1203+0.05 \%$ mucin.

755. tyrobutyricum, ATCC25755. The strain was cultured in $37^{\circ} \mathrm{C}, 10 \% \mathrm{H}_{2}, 5 \% \mathrm{CO}_{2}$ and $85 \% \mathrm{~N}_{2}$, medium 2107.

756. butyricum, ATCC19398. The strain was cultured in $37^{\circ} \mathrm{C}, 10 \% \mathrm{H}_{2}, 5 \% \mathrm{CO}_{2}$ and $85 \% \mathrm{~N} 2$, medium 2107 .

Experimental Design: A total of three in vitro experiments (Experiments 1-3) were performed; details of the experimental replication are provided in the corresponding figure legends.

For in vitro Experiment 1, culture medium (CM) of bacterial growth condition was prepared with the following protocol: fresh faeces pellets from normal donor mice (50-100 mg in $1 \mathrm{ml} \mathrm{PBS}$ ) were vortexed for $30 \mathrm{~min}$ and centrifuged for $10 \mathrm{~min}$ at $1000 \mathrm{~g}$ to remove faecal fibrous solids. The collected bacteriacontained supernatants $(500 \mu \mathrm{l})$ were supplemented with LB medium $(200 \mu \mathrm{l})$ and treated with sennoside A $(1 \mathrm{mg} / \mathrm{ml})$ for a stationary incubation at $37^{\circ} \mathrm{C}$ for 4 hours under anaerobic atmosphere. Then, the samples were centrifuged for $10 \mathrm{~min}$ at $15000 \mathrm{~g}$ to collect bacteria-free supernatants before being subjected to vacuum centrifugal concentration for $\sim 8-12 \mathrm{~h}$. Then, the samples were resuspended in $1 \mathrm{~mL}$ of corresponding strain medium (100\% SenA-CM). A ten point concentration (\%SenA-CM) using 2-fold serial dilutions was prepared.

For in vitro Experiments 2\&3, all species were evaluated in different concentrations of compound prototypes (sennoside A and rhein).

All species were cultured in a micro-anaerobic incubation system (ELECTROTEK, AW500SG/TG) and maintained at $37^{\circ} \mathrm{C}$ in a humidified incubator containing $10 \% \mathrm{H}_{2}, 5 \% \mathrm{CO}_{2}$ and $85 \% \mathrm{~N}_{2}$. Absorbance values were measured at $600 \mathrm{~nm}$ (A600) at intervals of $12 \mathrm{hr}$ over $36 \mathrm{hr}$ except for Akkermansia muciniphila, for which the absorbance was measured over $72 \mathrm{hr}$, owing to its relatively slow growth.

Notes. The colour of compounds (sennoside A and rhein) seriously affects the absorbance value of OD600. Therefore, the drug solution of different concentrations without bacteria (SenA, Sen A-CM, rhein) was used as a blank (see Table S1 for compositions of all growth media used in this study).

\section{Quantification of Short-Chain Fatty Acids by GC-MS}

Faecal samples stored at $-80^{\circ} \mathrm{C}$ were used to quantify short-chain fatty acids (SCFAs), including acetic acid, propionic acid, butyric acid, valeric acid, isovaleric acid, and isobutyric acid. Samples were first thawed on wet ice. Then, an equivalent amount of $0.05 \mathrm{M} \mathrm{NaOH}$ was added $(100 \mu \mathrm{l}$ per $100 \mathrm{mg}$ of material) to caecal contents ( $\geq 0.05 \mathrm{~g}$ ), and the samples were thoroughly homogenized by vortexing for $20 \mathrm{~min}$. Supernatants $(500 \mu \mathrm{l})$ were prepared by reconstituting all caecal contents of each animal with 10 $\mu \mathrm{l}$ of an internal standard (d3-caproic acid, $500 \mu \mathrm{g} / \mathrm{ml}$ ), followed by centrifugation at $13000 \mathrm{rpm}$ for 10 
min at $4^{\circ} \mathrm{C}$. The mixture (supernatants-internal standard- $\mathrm{NaOH}$ ) was kept on ice or frozen until quantification of the SCFAs by GC-MS, at which point the mixture was transferred to a glass tube, mixed with Milli-Q water and vortexed for 30 seconds. The SCFAs were extracted through the addition of $300 \mu \mathrm{L}$ of 1-propanol, $200 \mu \mathrm{l}$ of pyridine and $150 \mu \mathrm{l}$ of propyl chlorocarbonate by vortex mixing for $1 \mathrm{~min}$. Then, $500 \mu \mathrm{l}$ of hexyl hydride was added to the mixture, and it was subsequently vortexed for $1 \mathrm{~min}$ and centrifuged at $3000 \mathrm{rpm}$ for $5 \mathrm{~min}$. The extracted samples were measured using an ISQ GC-MS (Thermo Fisher Scientific, Waltham, MA, USA) as described previously with a TR-SQC capillary column $(15 \mathrm{~m} \times 0.25$ $\mathrm{mm})$ ). Helium was used as the carrier gas, and injections $(1 \mu \mathrm{l})$ were performed in split mode (10:1 split). The oven temperature was set at $50^{\circ} \mathrm{C}$ and maintained for $10 \mathrm{~min}$, then it was increased to $70^{\circ} \mathrm{C}$ at $10^{\circ} \mathrm{C} / \mathrm{min}$, increased to $85^{\circ} \mathrm{C}$ at $3^{\circ} \mathrm{C} / \mathrm{min}$, increased to $110^{\circ} \mathrm{C}$ at $5^{\circ} \mathrm{C} / \mathrm{min}$, increased to $290^{\circ} \mathrm{C}$ at $30^{\circ} \mathrm{C} / \mathrm{min}$ and held at this temperature for $8 \mathrm{~min}$. The detector temperature was set at $290^{\circ} \mathrm{C}$, and the injector temperature was $260^{\circ} \mathrm{C}$. SCFA standards were mixtures of acetic acid, propionic acid, butyric acid, isobutyric acid, valeric acid, and isovaleric acid. All standards were purchased from Merck (Darmstadt, Germany). The concentrations of SCFAs were calculated by the standard curve method. The reported values were normalized according to the wet weight of the original faecal sample used.

\section{Metabolomics analysis.}

For faecal metabolomics, approximately $50 \mathrm{mg}$ of faecal content was collected for each mouse at the end of the experiment, and to that was added $0.8 \mathrm{ml}$ of ultrapure water containing $6 \mu \mathrm{g} 1,2-{ }^{13} \mathrm{C}_{2}$-myristic to act as an internal standard, which was followed by vortexing for 5 min and centrifugation for $10 \mathrm{~min}$ at $13000 \mathrm{~g}$. The supernatants were transferred to a new $1.5 \mathrm{ml}$ tube. Then, $400 \mu \mathrm{L}$ of supernatants were dried in a SpeedVac sample concentrator and combined with $60 \mu \mathrm{L}$ of methoxyamine hydrochloride in pyridine $(10 \mathrm{mg} / \mathrm{mL})$, vortexed for $3 \mathrm{~min}$ and shaken at $30^{\circ} \mathrm{C}$ for $90 \mathrm{~min}$. Sixty microlitres of BSTFA containing $1 \%$ TMCS was added to the sample and shaken at $30^{\circ} \mathrm{C}$ for another $60 \mathrm{~min}$. The mixture was then transferred to a sampler vial with a glass insert and was subjected to GC-MS analysis. Quality control (QC) samples were prepared by pooling aliquots of all the faecal samples and were processed using the same procedure that was used for the experimental samples. Analysis was performed on a TRACE 1310 gas chromatograph equipped with an AS 1310 autosampler connected to a TSQ 8000 triple quadrupole mass spectrometer (Thermo Fisher Scientific, Waltham, MA, USA), as described previously[44]. Helium was used as the carrier gas and was maintained at a constant flow of $1.2 \mathrm{~mL} / \mathrm{min}$. The oven temperature was initially maintained at $60^{\circ} \mathrm{C}$ for $1 \mathrm{~min}$, then was increased to $320^{\circ} \mathrm{C}$ at $20^{\circ} \mathrm{C} / \mathrm{min}$, and then was held constant for $5 \mathrm{~min}$. The transfer line temperature between the gas chromatograph and the mass spectrometer was set to $250^{\circ} \mathrm{C}$. Electron impact ionization was performed at $70 \mathrm{eV}$, with an ion source temperature of $280^{\circ} \mathrm{C}$. Mass spectra were acquired with a scan range of $50-$ $500 \mathrm{~m} / \mathrm{z}$ and a time range of 3.5-19 min. Raw data were acquired from Xcalibur 2.2 software (Thermo Fisher Scientific), and metabolites were identified through matching of their mass spectra against reference spectra in the NIST 2014 standard database built-in Xcalibur 2.2 software. Metabolic analyses were performed using MetaboAnalyst 4.0 (https://www.metaboanalyst.ca/). Differential metabolites were 
identified by fold change $>2$ and $p$ value $<0.05$. Pathway enrichment analysis was carried out based on the above differential metabolites.

\section{ELISA for Fecal Lipocalin}

Frozen fecal samples $\left(-80^{\circ} \mathrm{C}\right.$ stored) were used to determine the levels of fecal Lipocalin (LCN2). The assays were performed within 30 days of sample collection. The samples were prepared as mentioned previously[45], with a few modifications in the sample prepration protocol: fecal samples were thawed on wet ice and $40-70 \mathrm{mg}$ of samples were separated in fresh tubes, to which $0.6 \mathrm{~mL}$ of $1 \%$ (v/v) Tween 20 (Sigma-Aldrich, USA) prepared in PBS was added. To get a homogeneous suspension, the samples were vortexed for $20 \mathrm{~min}$. The suspension was then centrifuged at $4^{\circ} \mathrm{C}$ for $10 \mathrm{~min}$ at $14000 \mathrm{rpm}$. Next, the supernatant was carefully recovered and stored at $-20^{\circ} \mathrm{C}$ until the analysis. To measure the LCN2 levels, a mouse Mouse Lipocalin-2/NGAL DuoSet ELISA (R\&D systems, Cat.No.DY1857) was employed and the manufacturer's protocol was followed.

\section{Measurement of cytokines in serum.}

Cytokines and chemokines were measured a Luminex-100 system and the XMap Platform (Luminex Corporation). Each sample was run in duplicate in a 96-well micro titer plate using $25 \mu$ serum using MILLIPLEX MAP Mouse Cytokine/Chemokine Magnetic Bead Panel (MCYTOMAG-70K-10, Millipore)

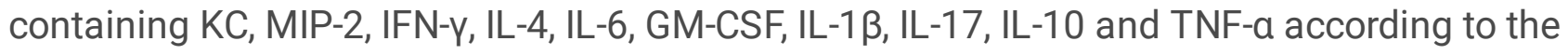
manufacturer's instructions. Quality control of each sample was performed, and a bead count of $<50$ was not used for analysis. The experimental design for the Luminex assay was carried out using PlateDesigner (platedesigner.net) with samples from the same patient being randomly allocated to a plate and well, guaranteeing that if technical confounders exist they can properly be adjusted for in the analysis steps[46].

\section{Flow cytometry analysis.}

Cells were isolated from mesenteric lymph nodes (mLNs), and then they were passed through $100 \mu \mathrm{m}$ cell strainers. Cells were counted and then were stimulated using Cell stimulation Cocktail plus transport inhibitors 500× (eBioscience $₫$ cat. no. 00-4975-93) for 4hr in complete RPMI1640 containing $10 \%$ FBS. After stimulation, cells were harvested, fixed using IC fixation buffer and permeabilized using $1 \times$ permeabilization buffer (eBioscience). The following antibody clones and staining reagents were used: anti-CD3-APC (eBioscience, 17-0032-82), anti-CD4-FITC (eBioscience, cat. no. MA5-17443), IL-17A-PerCPCyanine5.5 (eBioscience, 45-7177-82), IL-4-PE-Cy7 (8D4-8) (eBioscience, cat. no. 25-7049), and IFN gamma PE (XMG1.2) (eBioscience, cat. no. 12-7311-82). Single cell suspensions were examined using BD Accuri C6, and the data were analysed using C6 software and FlowJo software.

\section{Bacterial quantification by qPCR.}

In addition to $16 \mathrm{~S}$ rDNA sequencing of the relative abundance of Akkermansia muciniphila, phylotypespecific bacterial primers were designed as a second approach for absolute quantification of faecal 
samples. Total bacterial DNA was isolated from weighed faeces using an E.Z.N.A. Stool DNA Kit (Omega Bio-tek, USA). qPCR on a 7500 Sequence Detector (Applied Biosystems, CA, USA) was used to calculate the number of $A$. municiphila. DNA was then subjected to quantitative PCR using ChamQ SYBR qPCR Master Mix (Low ROX Premixed) (Vazyme, Shanghai, China) with the following primers: Amuc_1599F (GACCGGCATGTTCAAGCAGACT) and Amuc_1599R (AAGCCGCATTGGGATTATTTGTT)[45]. Standard curves for quantification consisted of ten-fold serial dilutions in the range of 108-101 copies of the 16S rRNA gene of the stool samples. The results are expressed as the number of bacteria per $\mathrm{mg}$ of stool using a standard curve.

\section{Statistical analysis}

Unless otherwise stated in the individual method sections above, statistical analysis was performed using GraphPad Prism V.7.0a. The results represent data from multiple independent experiments. Data are expressed as the mean \pm standard deviation (SD) or standard error of the mean (SEM). The data were analysed using two-tailed Student's $t$ tests (for two groups) and one-way analysis of variance (ANOVA) (for multiple groups). Statistically significant differences are shown with asterisks as follows: ${ }^{\star} p<0.05$, $* * p<0.01$, and $* * * p<0.001$.

\section{Declarations}

\section{Ethics Committee Approval and Patient Consent}

All experimental protocols were approved by the Animal Care and Use Committee of Nanjing University of Chinese Medicine (Nanjing, China) and conducted conforming to the Guidelines for the Care and Use of Laboratory Animals (ACU170904).

\section{Consent for publication}

Not applicable.

\section{Data Availability}

The datasets generated during and/or analyzed during the current study are available from the corresponding author on reasonable request. All 16S rRNA gene sequencing reads data has been deposited to the National Center for Biotechnology Information's Sequence Read Archive under accession number PRJNA663681.

\section{Competing Interest}

The authors declare that they have no competing interests

\section{Funding}


This work was financially supported by National Natural Science Foundation of China (81961128020, 82004124, 81973734, 81673795)

\section{Authors' contributions}

ZHW, JWW and YL designed the study protocol and supervised all parts of the project. PLS and YY conducted animal experiments. PC performed the molecular biology experiments. LT and YLS did the bioinformatics analyses in close collaboration with ZHW drafted the first versions. YLS and ZGS contributed to text revision and discussion. All authors read and approved the final manuscript.

\section{Acknowledgements}

The authors thank Prof. Jinjun Shan (Department of Pediatrics, Affiliated Hospital of Nanjing University of Chinese Medicine) for the quantification of Short-Chain Fatty Acids. The authors would also like to thank Drs Lei Chen (Human Genetics Institute of New Jersey, Rutgers University) for her comments on the manuscript

This project was supported in part by Jiangsu Province Traditional Chinese Medicine Leading Talents Program (SLJ0229).

\section{References}

[1] Ford, Suares.Effect of laxatives and pharmacological therapies in chronic idiopathic constipation: systematic review and meta-analysis.Gut.2011; 60: 209-18.

[2] Malik, Müller.Anthraquinones As Pharmacological Tools and Drugs.Med Res Rev.2016; 36: 705-48.

[3] Dufour, Gendre.Ultrastructure of mouse intestinal mucosa and changes observed after long term anthraquinone administration.Gut.1984; 25: 1358-63.

[4] Mori, Sugie, Niwa, Yoshimi, Tanaka, Hirono.Carcinogenicity of chrysazin in large intestine and liver of mice.Jpn. J. Cancer Res.1986; 77: 871-76.

[5] Siegers, von, Otte, Schneider.Anthranoid laxative abuse-a risk for colorectal cancer.Gut.1993; 34: 1099101.

[6] Mereto, Ghia, Brambilla.Evaluation of the potential carcinogenic activity of Senna and Cascara glycosides for the rat colon.Cancer Lett.1996; 101: 79-83.

[7] NTP Toxicology and Carcinogenesis Studies of EMODIN (CAS NO. 518-82-1) Feed Studies in F344/N Rats and B6C3F1 Mice.Natl Toxicol Program Tech Rep Ser.2001; 493: 1-278.

[8] Martens, Neumann, Desai.Interactions of commensal and pathogenic microorganisms with the intestinal mucosal barrier.Nat. Rev. Microbiol.2018; 16: 457-70. 
[9] Cai, Cheng, Chen, Xu, Ding, Gu.Interactions of commensal and pathogenic microorganisms with the mucus layer in the colon.Gut Microbes.2020; 11: 680-90.

[10] Abreu.Toll-like receptor signalling in the intestinal epithelium: how bacterial recognition shapes intestinal function.Nat. Rev. Immunol.2010; 10: 131-44.

[11] Grivennikov, Wang, Mucida, Stewart, Schnabl, Jauch, Taniguchi, Yu, Osterreicher, Hung, Datz, Feng, Fearon, Oukka, Tessarollo, Coppola, Yarovinsky, Cheroutre, Eckmann, Trinchieri, Karin.Adenoma-linked barrier defects and microbial products drive IL-23/IL-17-mediated tumour growth.Nature.2012; 491: 25458.

[12] Gallimore, Godkin.Epithelial barriers, microbiota, and colorectal cancer.N. Engl. J. Med.2013; 368: 282-84.

[13] Chen, Pitmon, Wang.Microbiome, inflammation and colorectal cancer.Semin. Immunol.2017; 32: 4353.

[14] Li, Limenitakis, Fuhrer, Geuking, Lawson, Wyss, Brugiroux, Keller, Macpherson, Rupp, Stolp, Stein, Stecher, Sauer, McCoy, Macpherson.The outer mucus layer hosts a distinct intestinal microbial niche.Nat Commun.2015; 6: 8292.

[15] van Gorkom BA, de Vries EG, Karrenbeld, Kleibeuker.Review article: anthranoid laxatives and their potential carcinogenic effects.Aliment. Pharmacol. Ther.1999; 13: 443-52.

[16] Van der Sluis M, De Koning BA, De Bruijn AC, Velcich, Meijerink, Van Goudoever JB, Büller, Dekker, Van Seuningen I, Renes, Einerhand.Muc2-deficient mice spontaneously develop colitis, indicating that MUC2 is critical for colonic protection.Gastroenterology.2006; 131: 117-29.

[17] Schroeder, GMH, Ståhlman, Arike, MEV, Hansson, Bäckhed.Bifidobacteria or Fiber Protects against Diet-Induced Microbiota-Mediated Colonic Mucus Deterioration.Cell Host Microbe.2018; 23: 27-40.e7.

[18] Wei, Yang, Rey, Ridaura, Davidson, Gordon, Semenkovich.Fatty acid synthase modulates intestinal barrier function through palmitoylation of mucin 2.Cell Host Microbe.2012; 11: 140-52.

[19] Okumura, Kurakawa, Nakano, Kayama, Kinoshita, Motooka, Gotoh, Kimura, Kamiyama, Kusu, Ueda, Wu, lijima, Barman, Osawa, Matsuno, Nishimura, Ohba, Nakamura, lida, Yamamoto, Umemoto, Sano, Takeda.Lypd8 promotes the segregation of flagellated microbiota and colonic epithelia.Nature.2016; 532: $117-21$.

[20] Tramontano, Andrejev, Pruteanu, Klünemann, Kuhn, Galardini, Jouhten, Zelezniak, Zeller, Bork, Typas, Patil.Nutritional preferences of human gut bacteria reveal their metabolic idiosyncrasies.Nat Microbiol.2018; 3: 514-22. 
[21] Parks, Tyson, Hugenholtz, Beiko.STAMP: statistical analysis of taxonomic and functional profiles.Bioinformatics.2014; 30: 3123-24.

[22] El, Armougom, Gordon, Raoult, Henrissat.The abundance and variety of carbohydrate-active enzymes in the human gut microbiota.Nat. Rev. Microbiol.2013; 11: 497-504.

[23] Yamada, Hino, lijima, Genda, Aoki, Nagata, Han, Hirota, Kinashi, Oguchi, Suda, Furusawa, Fujimura, Kunisawa, Hattori, Fukushima, Morita, Hase.Mucin O-glycans facilitate symbiosynthesis to maintain gut immune homeostasis.EBioMedicine.2019; 48: 513-25.

[24] Li, Yi, Katiraei, Kooijman, Zhou, Chung, Gao, van den Heuvel JK, Meijer, JFP, Heijink, Giera, van Dijk K, Groen, PCN, Wang.Butyrate reduces appetite and activates brown adipose tissue via the gut-brain neural circuit.Gut.2018; 67: 1269-79.

[25] Fachi, Felipe, Pral, da, Corrêa, de Andrade MCP, da, Basso, NOS, de Sales E Souza ÉL, Dos, SES, Thomas, Setubal, Magalhães, Forti, Candreva, Rodrigues, de Jesus MB, Consonni, ADS, Varga-Weisz, MAR.Butyrate Protects Mice from Clostridium difficile-Induced Colitis through an HIF-1-Dependent Mechanism.Cell Rep.2019; 27: 750-61.e7.

[26] Yusung, Braun.Molecular mimicry, inflammatory bowel disease, and the vaccine safety debate.BMC Med.2014; 12: 166.

[27] Brown, Round.Friends in Low Places: Intestinal Commensals Limit Colitis through Molecular Mimicry.Cell.2017; 171: 503-05.

[28] Chassaing, Koren, Goodrich, Poole, Srinivasan, Ley, Gewirtz.Dietary emulsifiers impact the mouse gut microbiota promoting colitis and metabolic syndrome.Nature.2015; 519: 92-96.

[29] Koh, De Vadder F, Kovatcheva-Datchary, Bäckhed.From Dietary Fiber to Host Physiology: Short-Chain Fatty Acids as Key Bacterial Metabolites.Cell.2016; 165: 1332-45.

[30] Andrews, Gamache, Weingarten, Haimes.Intraspinal extension of an air cyst of the lung: case report.Neurosurgery.1989; 24: 414-17.

[31] Manna, Tanaka, Krausz, Haznadar, Xue, Matsubara, Bowman, Fearon, Harris, Shah, Gonzalez.Biomarkers of coordinate metabolic reprogramming in colorectal tumors in mice and humans.Gastroenterology.2014; 146: 1313-24.

[32] Chassaing, Ley, Gewirtz.Intestinal epithelial cell toll-like receptor 5 regulates the intestinal microbiota to prevent low-grade inflammation and metabolic syndrome in mice.Gastroenterology.2014; 147: 136377.e17.

[33] Arqués, Chicote, Puig, Tenbaum, Argilés, Dienstmann, Fernández, Caratù, Matito, Silberschmidt, Rodon, Landolfi, Prat, Espín, Charco, Nuciforo, Vivancos, Shao, Tabernero, Palmer.Tankyrase Inhibition 
Blocks Wnt/ $\beta$-Catenin Pathway and Reverts Resistance to PI3K and AKT Inhibitors in the Treatment of Colorectal Cancer. Clin. Cancer Res. 2016, 22, 644-56.

[34] Tenbaum, Ordóñez-Morán, Puig, Chicote, Arqués, Landolfi, Fernández, Herance, Gispert, Mendizabal, Aguilar, Ramón, Schwartz, Vivancos, Espín, Rojas, Baselga, Tabernero, Muñoz, Palmer.ß-catenin confers resistance to PI3K and AKT inhibitors and subverts FOXO3a to promote metastasis in colon cancer. Nat. Med. 2012, 18, 892-901.

[35] Sharkey, Beck, McKay.Neuroimmunophysiology of the gut: advances and emerging concepts focusing on the epithelium.Nat Rev Gastroenterol Hepatol.2018; 15: 765-84.

[36] Yu, Pardoll, Jove.STATs in cancer inflammation and immunity: a leading role for STAT3.Nat. Rev. Cancer.2009; 9: 798-809.

[37] Dammann, Khare, Gasche.Tracing PAKs from GI inflammation to cancer.Gut.2014; 63: 1173-84.

[38] Louis, Hold, Flint.The gut microbiota, bacterial metabolites and colorectal cancer.Nat. Rev. Microbiol.2014; 12: 661-72.

[39] Khandekar, Cohen, Spiegelman.Molecular mechanisms of cancer development in obesity.Nat. Rev. Cancer.2011; 11: 886-95.

[40] Yu, Lee, Herrmann, Buettner, Jove.Revisiting STAT3 signalling in cancer: new and unexpected biological functions.Nat. Rev. Cancer.2014; 14: 736-46.

[41] Li, Sun, Li, Zhang, Cui, Yun, Yang, Zhang, Meng, Wu, Duan, Yang, Wu, Sun, Zou, Chen.Probiotics Ameliorate Colon Epithelial Injury Induced by Ambient Ultrafine Particles Exposure.Adv Sci (Weinh).2019; 6: 1900972.

[42] Johansson, Hansson.Preservation of mucus in histological sections, immunostaining of mucins in fixed tissue, and localization of bacteria with FISH.Methods Mol. Biol.2012; 842: 229-35.

[43] Martinez, Dusserre, Wahli, Mermod.Synergistic transcriptional activation by CTF/NF-I and the estrogen receptor involves stabilized interactions with a limiting target factor.Mol. Cell. Biol.1991; 11: 2937-45.

[44] Li, Yang, Dai, Lin, Xie, He, Tao, Shan, Wang.Non-invasive urinary metabolomic profiles discriminate biliary atresia from infantile hepatitis syndrome.Metabolomics.2018; 14: 90.

[45] Desai, Seekatz, Koropatkin, Kamada, Hickey, Wolter, Pudlo, Kitamoto, Terrapon, Muller, Young, Henrissat, Wilmes, Stappenbeck, Núñez, Martens.A Dietary Fiber-Deprived Gut Microbiota Degrades the Colonic Mucus Barrier and Enhances Pathogen Susceptibility.Cell.2016; 167: 1339-53.e21. 
[46] Suprun, Suárez-Fariñas.PlateDesigner: a web-based application for the design of microplate experiments.Bioinformatics.2019; 35: 1605-07.

\section{Figures}


A
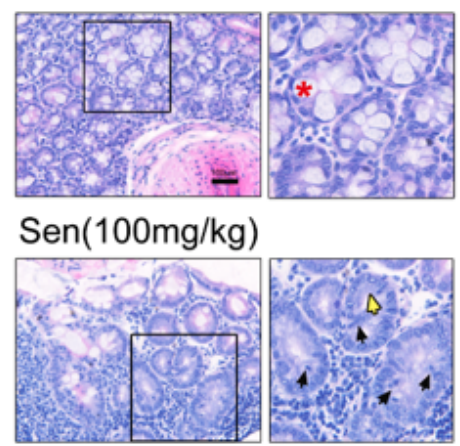

C

Sen $(\mathrm{mg} / \mathrm{kg}) 0$

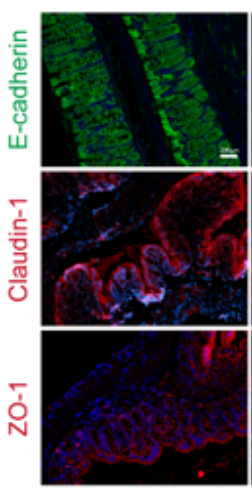

25
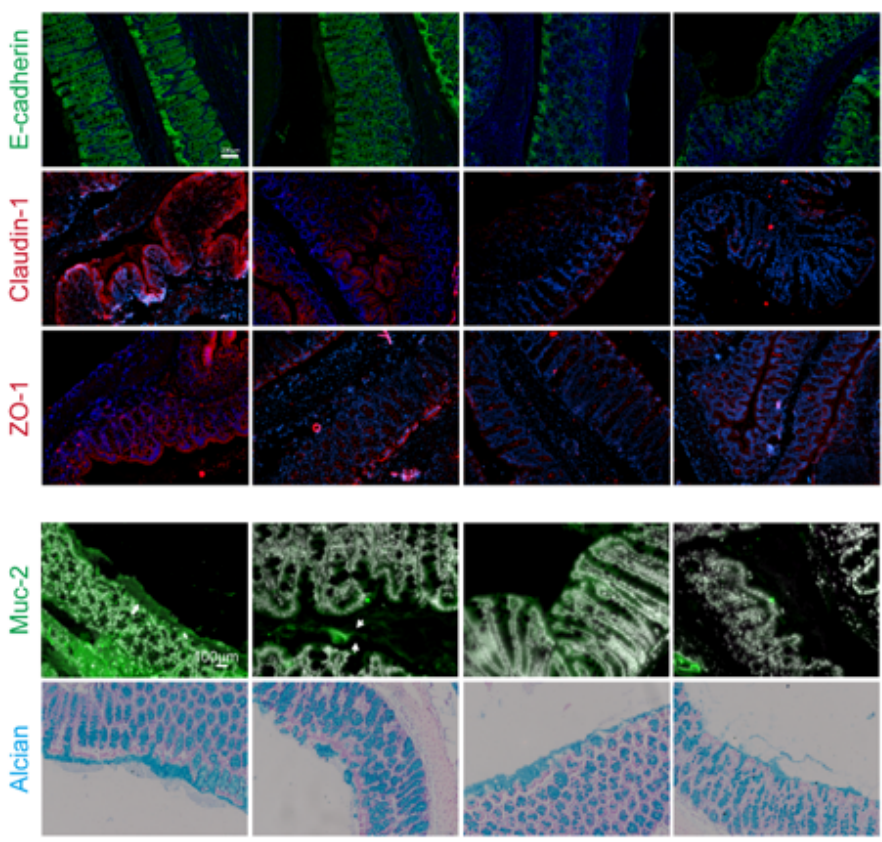

Sen $(25 \mathrm{mg} / \mathrm{kg})$

Sen $(50 \mathrm{mg} / \mathrm{kg})$

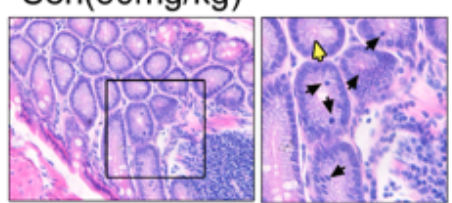

100
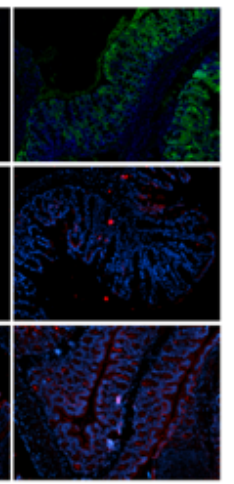

B
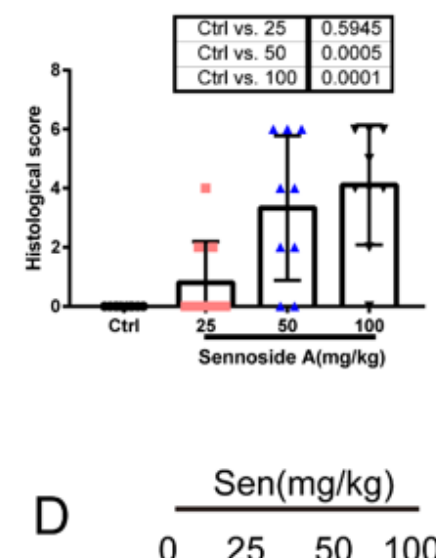

Claudin-1

GAPDH

G
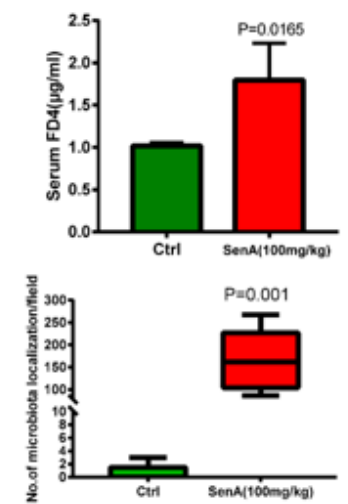

F FITC-Dextran/FD4/DAPI
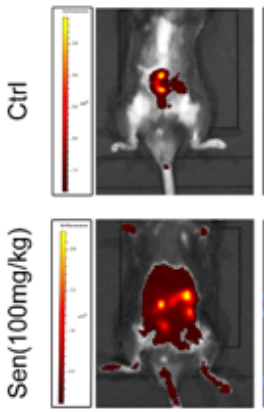
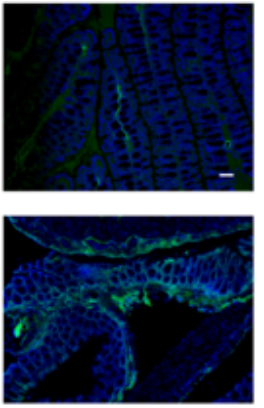

$\mathrm{H}$ EUB338/Muc-2/DAPI
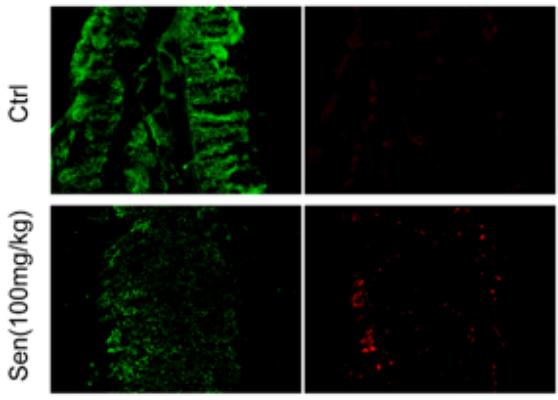

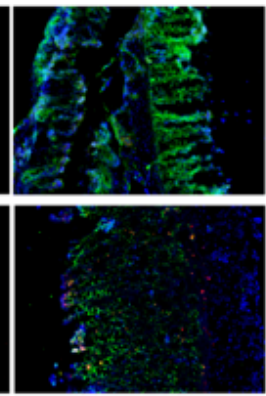

\section{Figure 1}

Sennoside A causes intestinal barrier injury and bacterial translocation. (A) Representative colon histological sections of sennoside A-treated mice and control mice (original magnification, $\times 400$ ). Red asterisks mark goblet cells filled with mucus. Arrows indicate major histopathological differences between groups: polymorphonuclear infiltration of the crypt (black arrows) and reduction of goblet cells (yellow arrows). (B) Histological score of mice treated with sennoside A from colonic tissue sections (day 
84). Each dot represents the histological score of one individual mouse. The horizontal bar represents the mean \pm SD. (C) Images of the ileal villi sections showing the tight and adherens junctions claudin-1 (red), E-cadherin (green), and ZO-1 (red) (magnification, $\times 200$ ), which are also seen in Figure S2. NCM-460 cell treatment with sennoside A. (D) Immunoblot of the cell-cell junction proteins claudin, E-cadherin and ZO-1 in whole- tissue lysates $(H)$ of colon IECs. (E) Immunofluorescence images of colonic thin sections stained with a-Muc2 antibody and DAPI (magnification, $\times 400$ ) and Alcian blue-stained colonic sections showing the inner mucus layer (magnification, $\times 200$ ). Please note that the outer mucus layer is less defined and is often not observed in fixed tissue sections. (F) In vivo detection of orally administered tracer (4-kDa FITC-dextran). Representative imaging of the animal abdomen is shown on the left, and fluorescence photography of the colonic epithelium is shown on the right (magnification, $\times 100)$. (G) Quantitation of serum FD4 permeability. $p=0.0165$ (with data presented as the mean $\pm S E M)$. $(H)$ Microscopy analysis of microbiota localization: EUB338I probe (red), MuC-2 (green) and DAPI (blue). Magnification, $\times 100$. (I) Number of bacterial localizations in the field. Data are the mean $\pm S E M, P=0.01$. 
A

Phylum

SenA(100mg/kg)

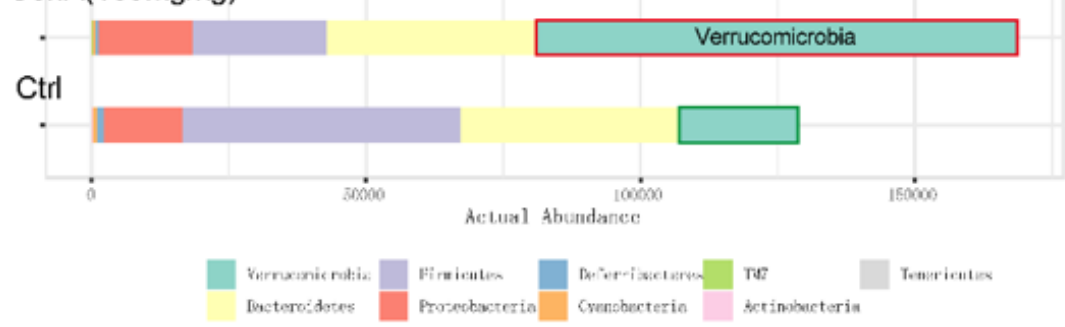

C DenA(100mg/kg)

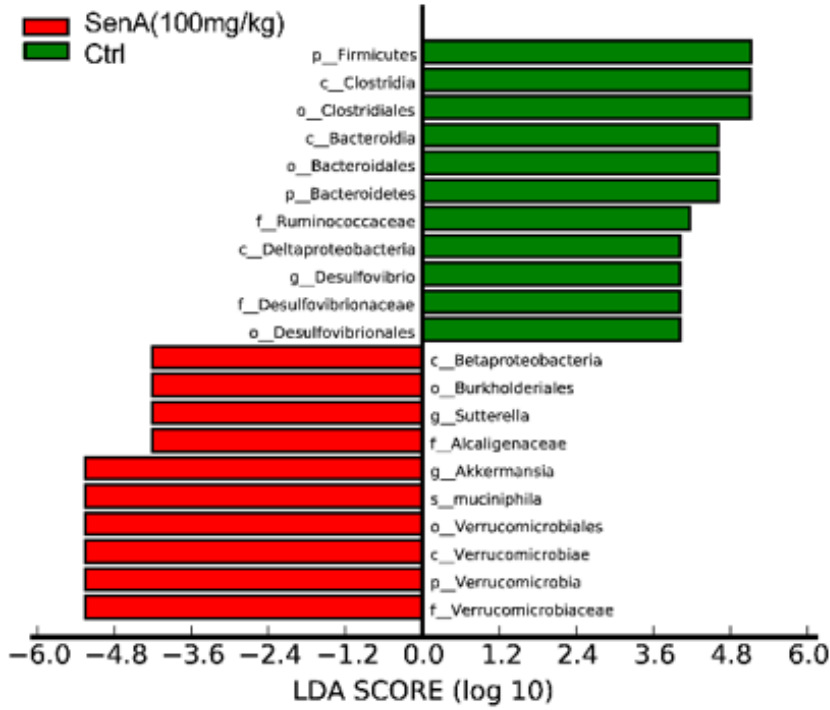

$\mathrm{F}$
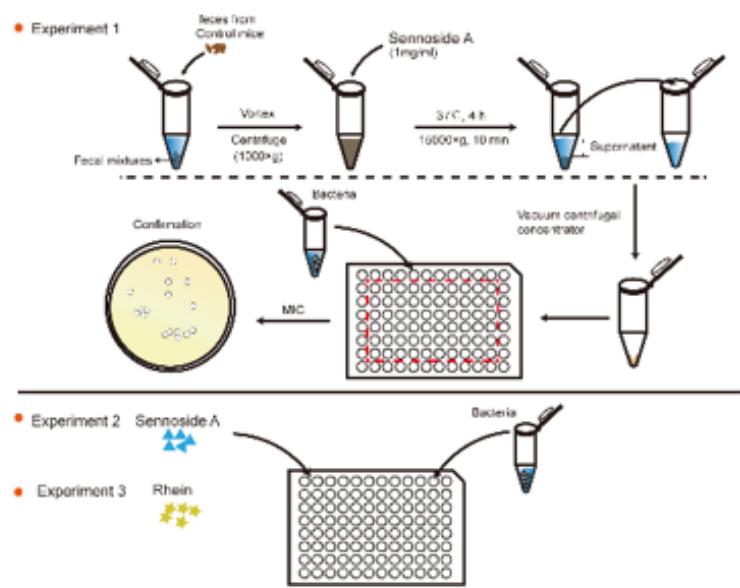

B

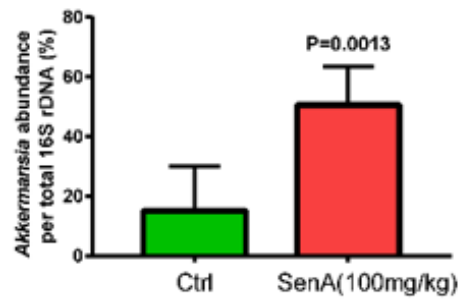

$\mathrm{D}$

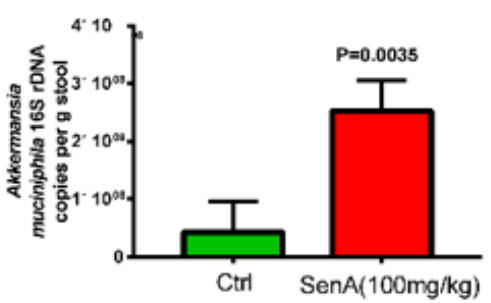

E

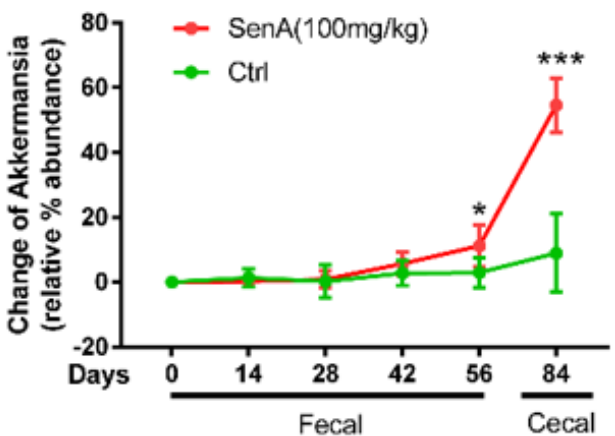

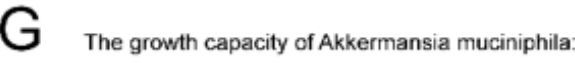

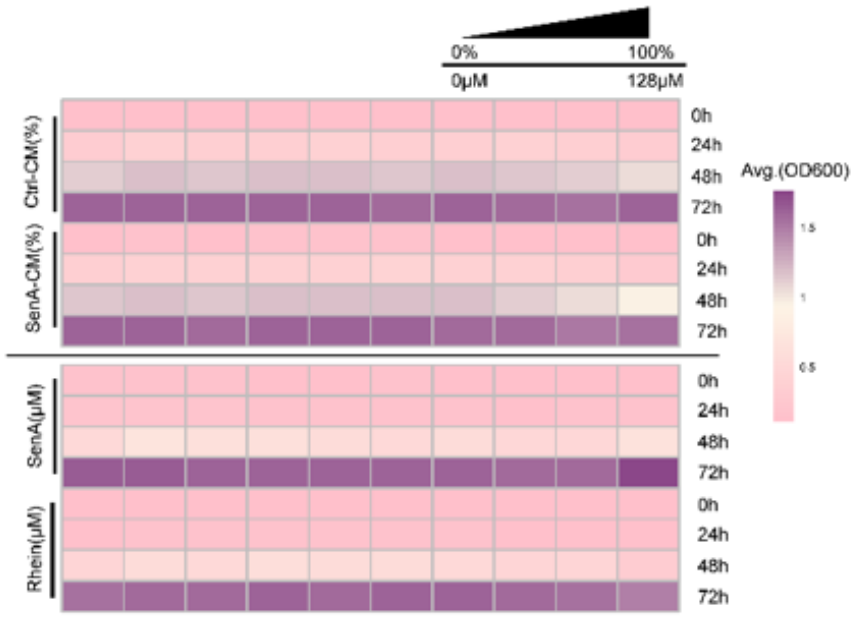

\section{Figure 2}

Sennoside A promotes enrichment of mucus-degrading bacteria. (A) Bacterial taxonomic profiling at the phylum level of intestinal bacteria from different mouse groups. (B) Relative abundance of Akkermansia. See also Figures S3. (C) 16S rDNA copies of Akkermansia muciniphila measured by quantitative PCR. (D) Linear discriminant analysis (LDA) scores derived from LEfSe analysis, showing the biomarker taxa at the genus level (LDA score) of $>4$ and a significance of $\mathrm{P}<0.05$ determined by the Wilcoxon signed-rank 
test. (E) Changes in relative Akkermansia abundance over time in mice oscillated for 14-day increments. Changes in the sennoside A treatment and control groups are shown for comparison. Asterisks indicate a statistically significant difference (SenA v.s. Ctrl) using Student's t test. (F) Experimental design of bacterial growth assays in vitro. See materials and methods for details. (G) Heat map showing the growth capacity of Akkermansia muciniphila strains in the respective media. The values shown are averages of OD600 data.

A

$\operatorname{Sen} \mathrm{A}(100 \mathrm{mg} / \mathrm{kg})$

Order

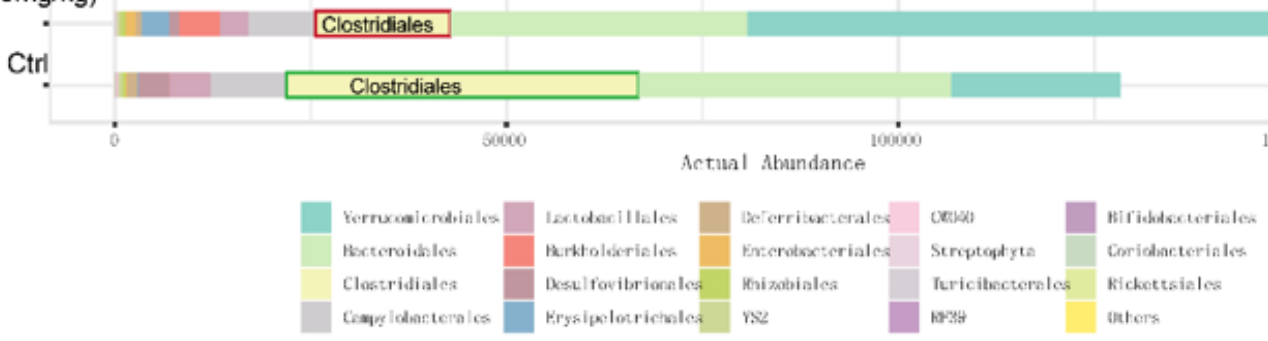

B

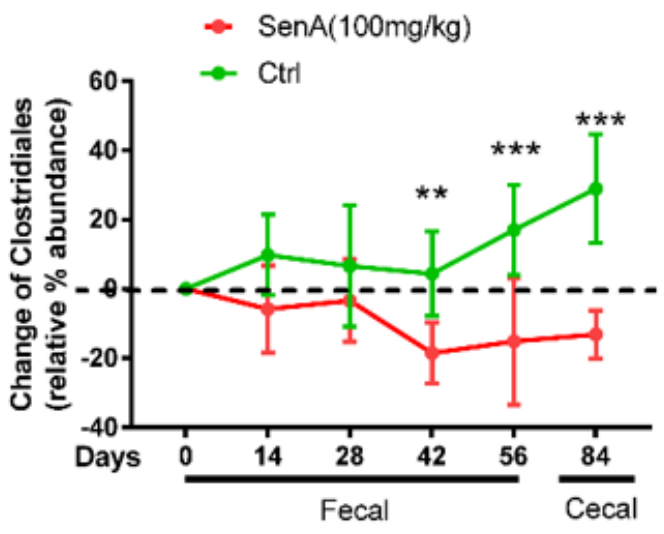

C

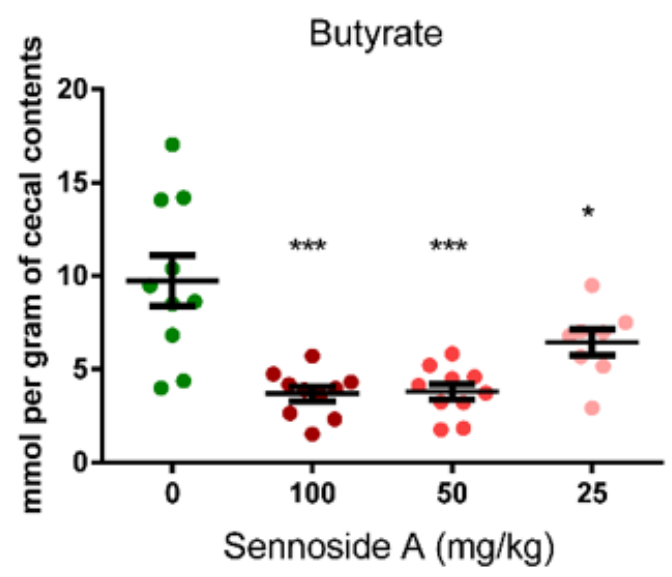

D

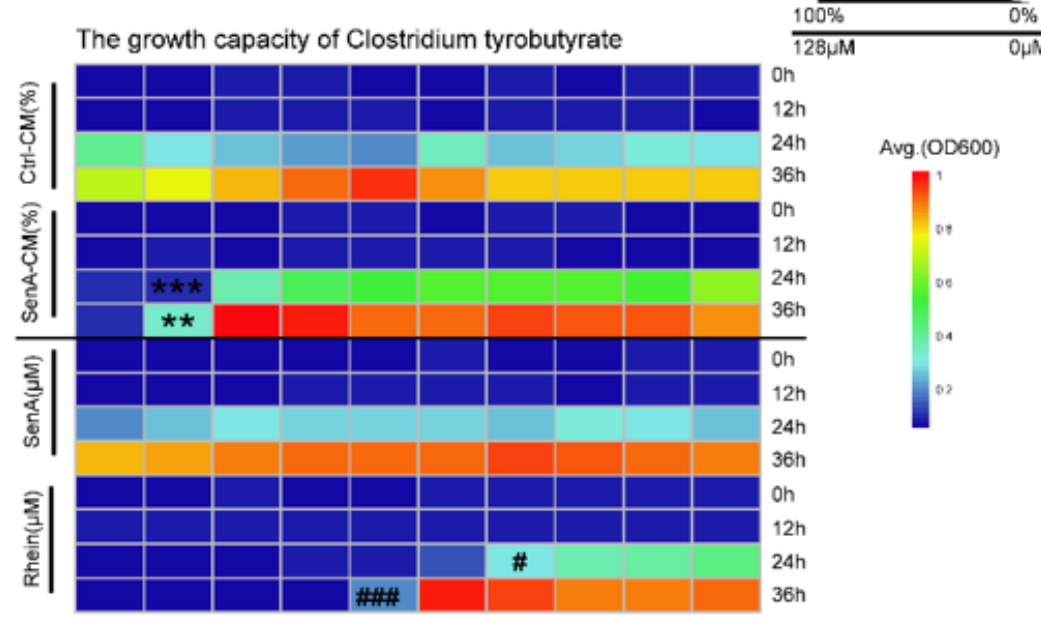

$E$

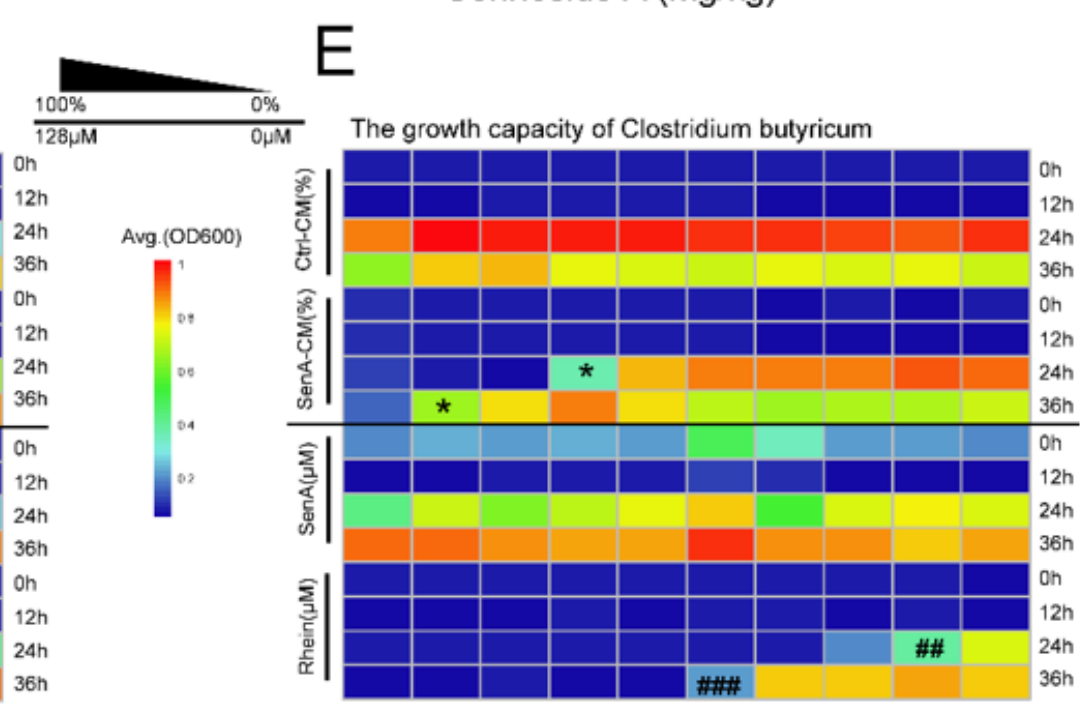

Figure 3

Sennoside A inhibited butyrate-producing bacterial growth. (A) Bacterial taxonomic profiling at the order level shows that sennoside A significantly inhibited the relative abundance of Clostridiales. (B) Stream 
plots exhibiting the faecal (over time) and caecal (end point) dynamic abundances of Clostridiales. ${ }^{\star *} p<0.01,{ }^{* \star} p<0.001$ versus Ctrl group at the same time points. (C) Concentrations of butyrate determined from caecal contents. Middle lines indicate the average of the individual measurements shown, and error bars represent the SEM. One-way analysis of variance. See other short chain fatty acids also measured by GC-MS in Figure S5. (D) Direct influence of sennoside A on butyrate production by Clostridiales. Influence of sennoside A and its intestinal metabolites on Clostridium tyrobutyricum (left panel) and Clostridium butyricum (right panel)
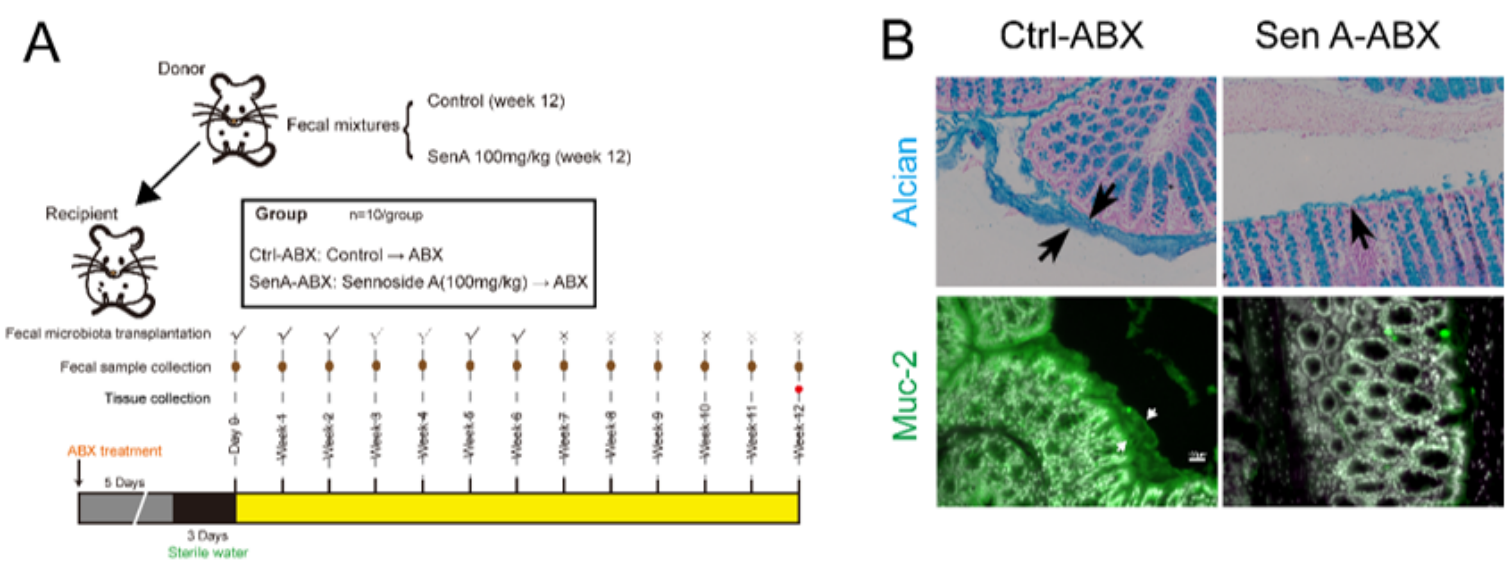

C
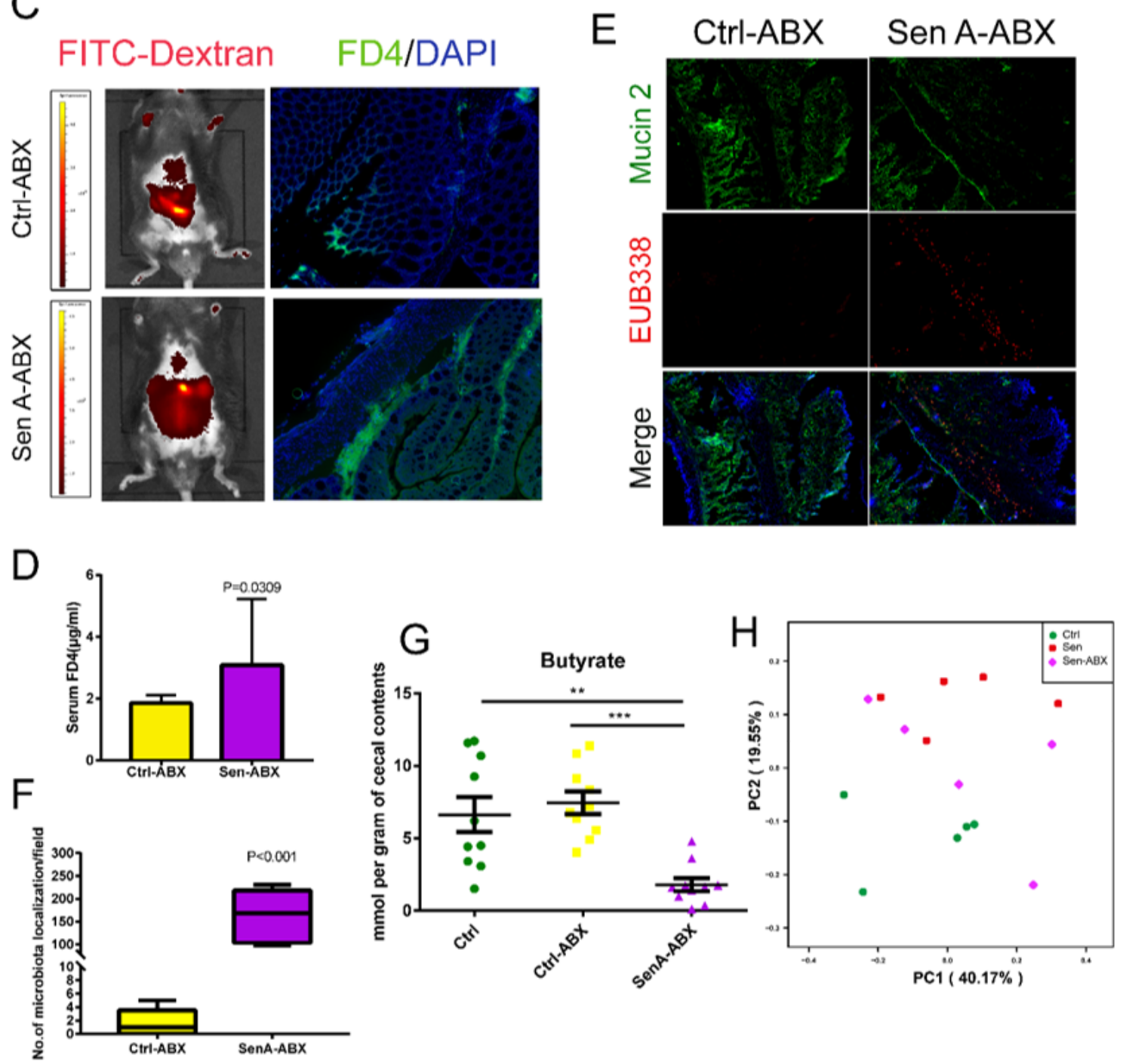


\section{Figure 4}

Sennoside A-induced gut barrier dysfunction is transferable by faecal transplantation. (A). Study design of the faecal transplant experiment. See Animal protocol 2 for detailed implementation strategies. (B) Disruption of the colonic mucus layer by faecal bacteria from sennoside A-treated mice (SenA-ABX) versus control mice (Ctrl-ABX), as detected by Muc2 immunofluorescence and Alcian blue staining. (C\&D) Imaging of live mice, colon sections and serum FD4 revealed that disruption of intestinal barrier integrity was observed in SenA-ABX mice. (E) Bacterial translocation across the intestinal barrier in SenA-ABX mice. Magnification, $\times 400$. (F) Bacteria counts in intracellular epithelial cells. (G) Measurement of SCFAs in the colon content of SenA-ABX and ctrl-ABX mice. See also Figure S6A-E. All error bars represent SEM. $\star \star \star * p<0.001 ; * \star p<0.01 ;{ }^{*} p<0.05$; ns, no significance. $(H)$ PCoA revealed that the SenA-ABX bacterial communities clustered separately from ctrl bacterial communities, and they were more similar to the SenA group. Each circle represents a single sample, coloured by group. 

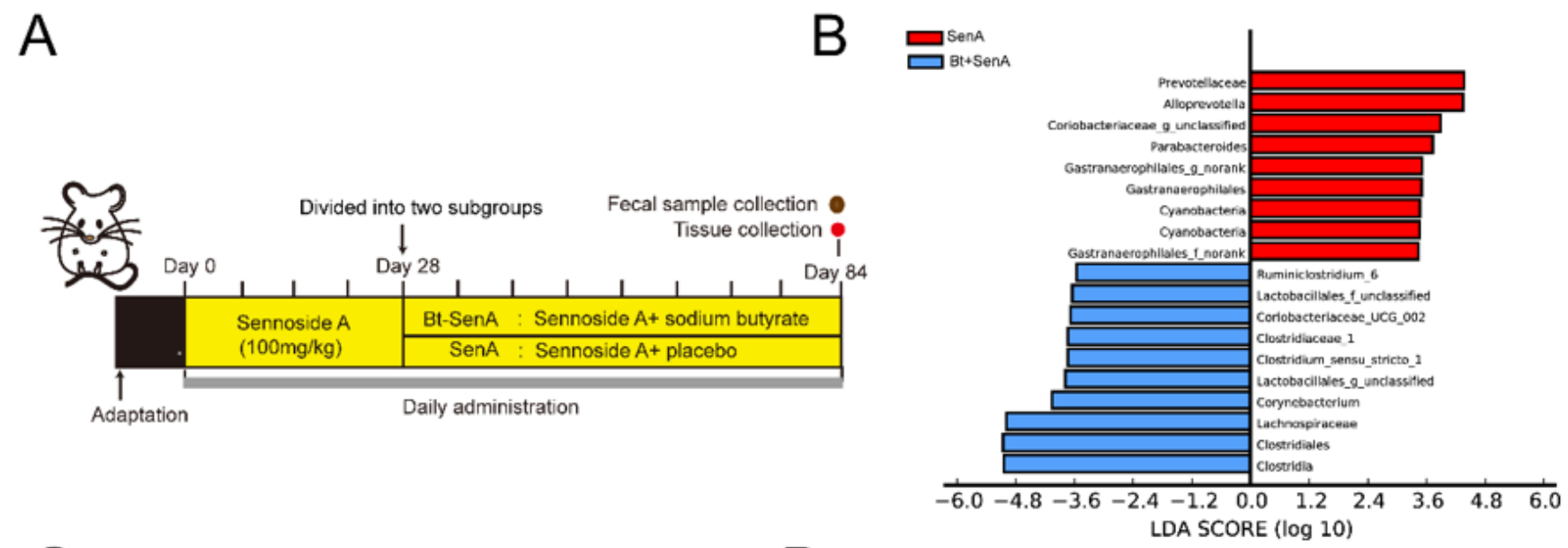
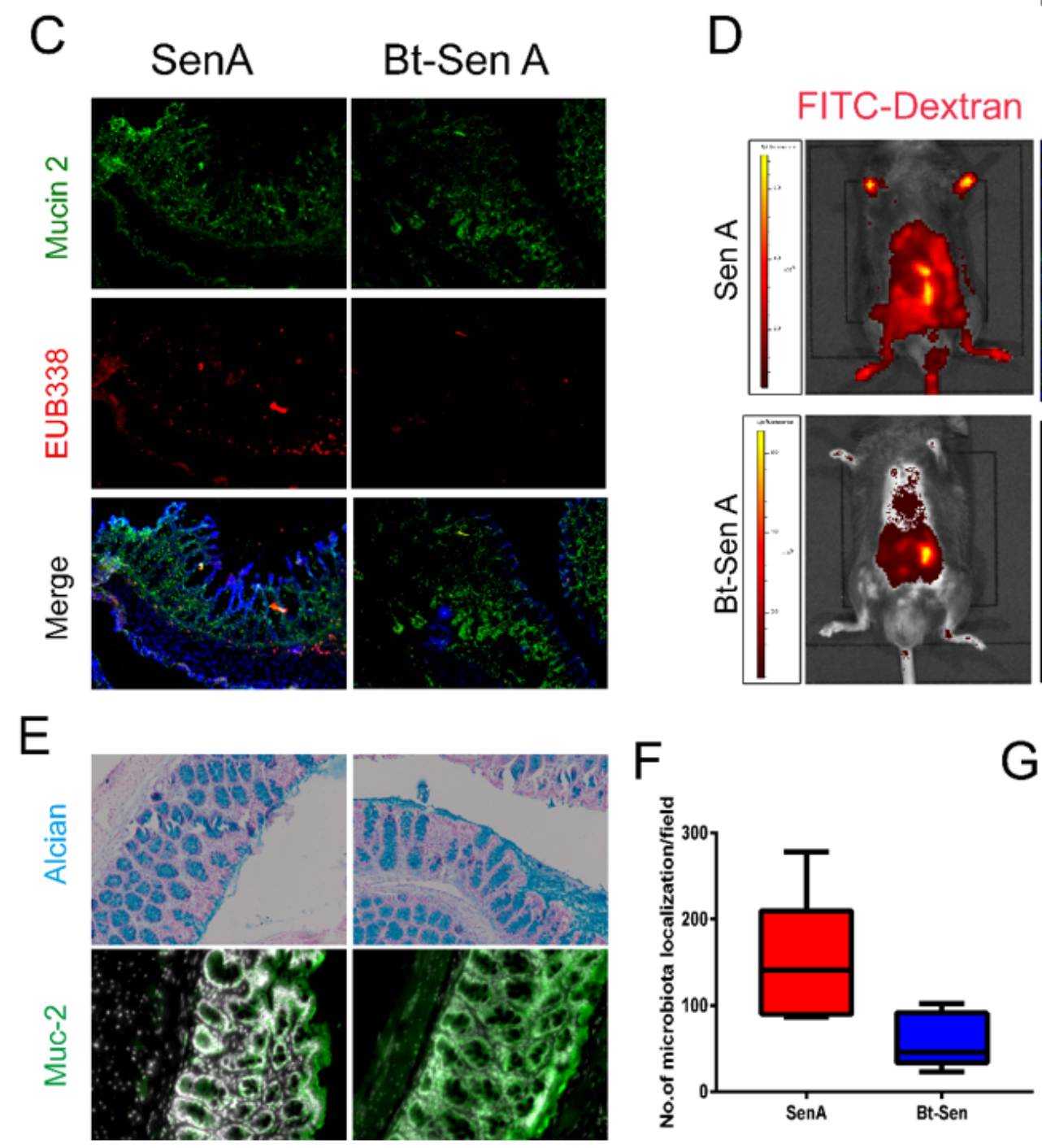

FITC-Dextran
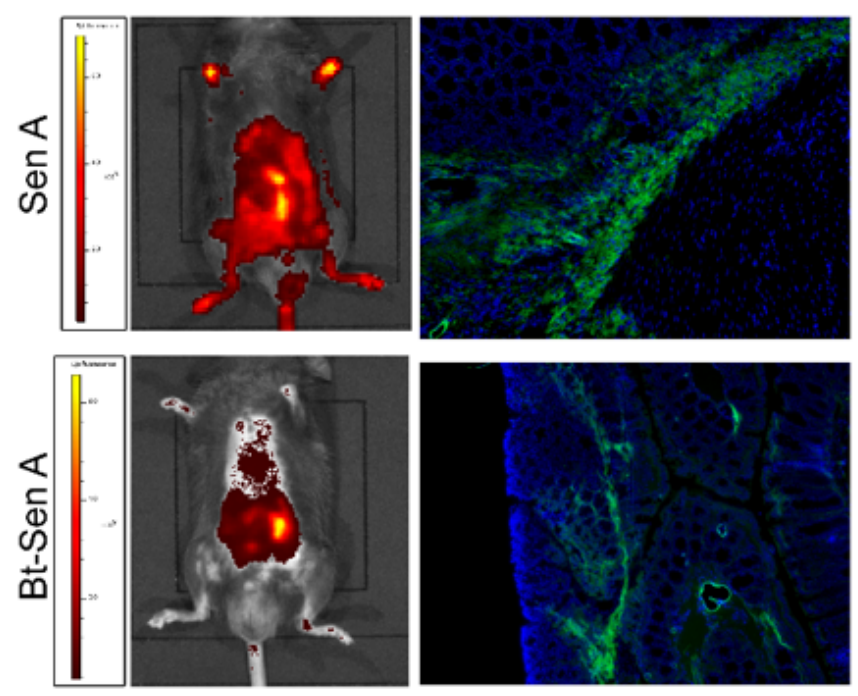

G

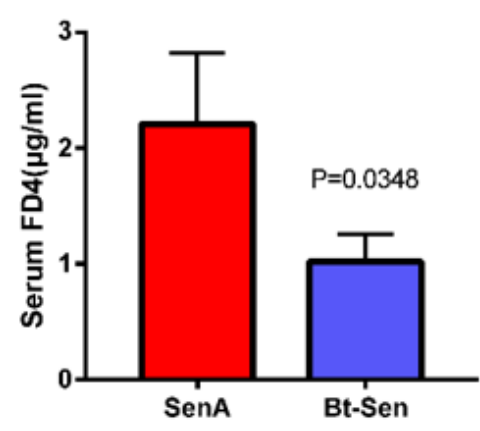

\section{Figure 5}

Oral administration of butyrate protects mice against sennoside A-induced damage. (A) Schematic of the butyrate supplement experimental design and collection protocol. See Animal protocol 3 for detailed implementation strategies. (B) LEfSe analysis identified the most differentially abundant taxa between the two groups. SenA-enriched taxa are indicated with a positive LDA score, and taxa enriched in Bt-SenA have a negative score. Only taxa meeting an LDA significant threshold of $>4$ are shown. (C) 
Representative in situ detection of bacteria in the SenA and Bt-SenA groups. Colon tissue sections were stained with DAPI (blue) and Muc2 (green) and probed with the universal eubacterial probe EUB338I (red). Original magnification, $\times 100$. (D) Evaluation of intestinal permeability. (E) Representative immunofluorescence (Muc-2) images (magnification, $\times 200$ ) and Alcian blue images (magnification, $\times 100)$. (F) Comparison of the total bacterial load in the field of the SenA and Bt-SenA groups. (G) Intestinal permeability was assessed with serum FD4.
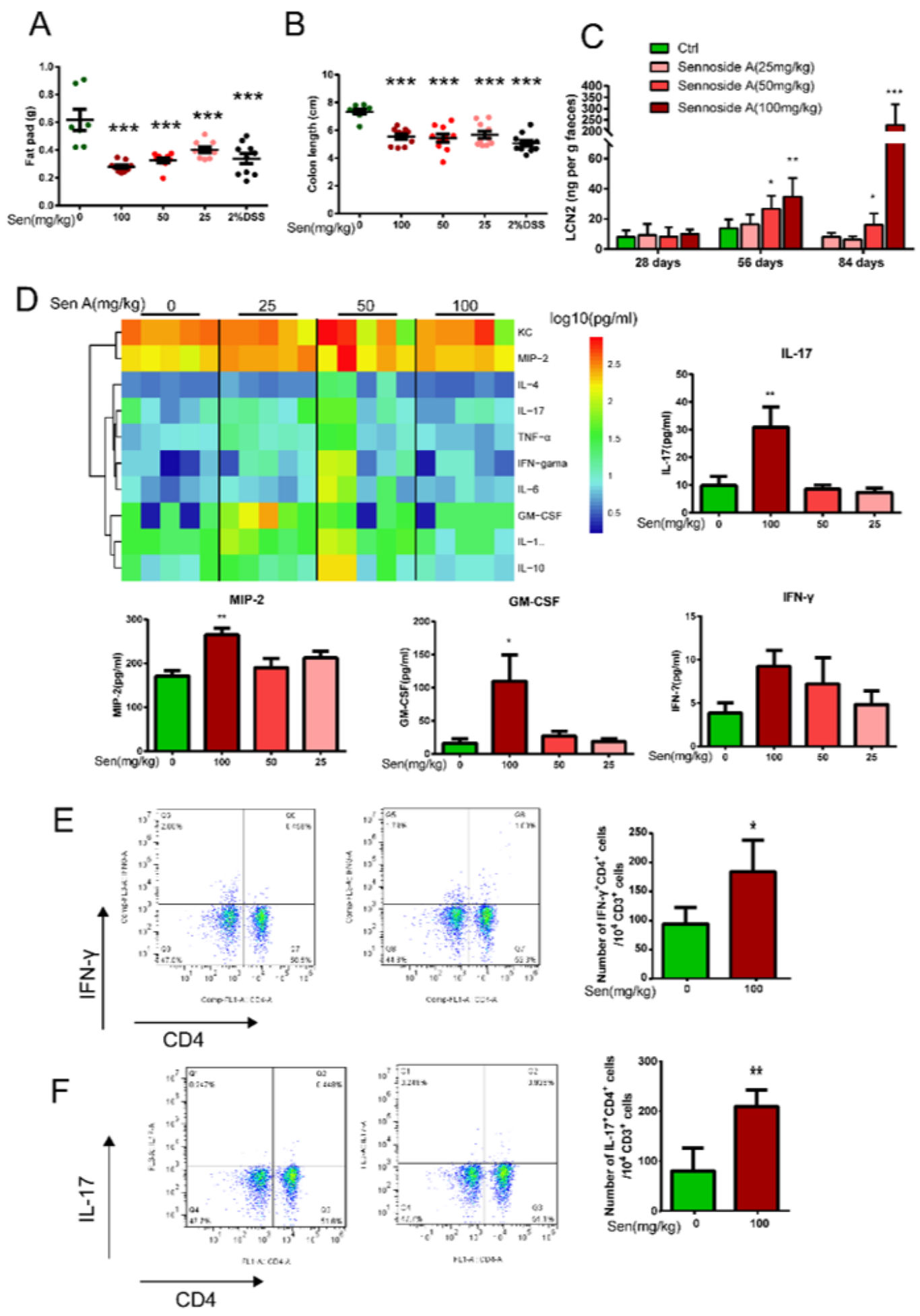

Figure 6 
Long-term sennoside A treatment induces chronic low-grade inflammation. (A) Visceral fat pad weight (epididymal white adipose tissue) and (B) colon length were measured after 12 weeks of treatment, as described in the 'Methods' section. (C) Faecal levels of the inflammatory marker LCN2 in each group at different time points ( $n=8-9$ mice per group). (D) Heatmap representation of ten specific cytokine levels in serum: CXCL1, CXCL2, IFN-ץ, IL-4, IL-6, GM-CSF, IL-1ß, IL-17, IL-10, and TNF-a. The colour of the heatmap in each row is based on $\log 10(\mathrm{pg} / \mathrm{mL})$ values $(n=4-5$ per group). Estimated Ismean and $95 \% \mathrm{Cl}$ are shown for selected cytokines. (D) Flow cytometric plots of IFN- $y$ and IL-17 expression on foetal and infant $\mathrm{CD} 4+$ cells in mesenteric lymph nodes. Statistical significance was measured by one-way ANOVA or ttest. The $p$ value is shown as ${ }^{*} p<0.05$, and ${ }^{* *} p<0.01$. 
A

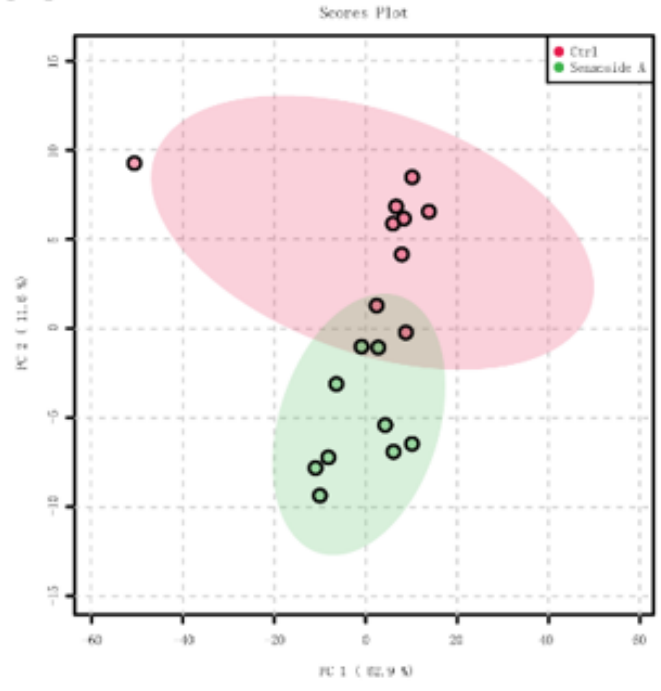

B

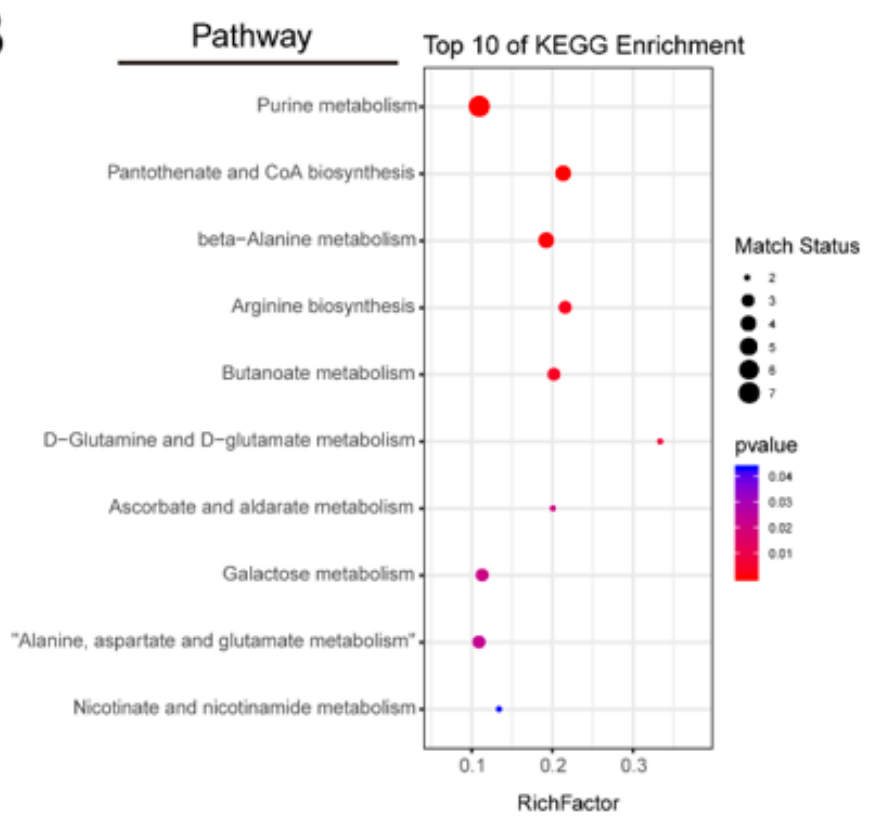

C
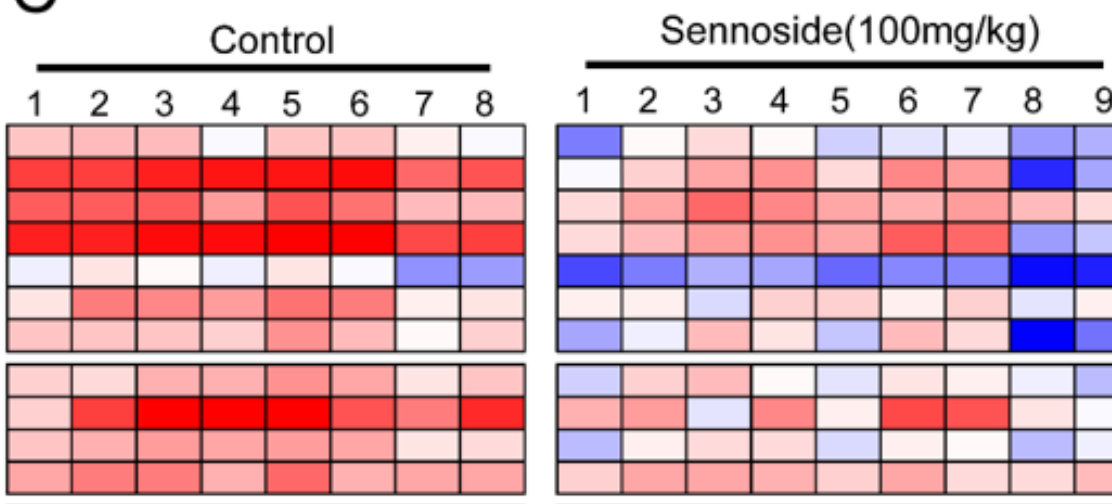

Metabolites

Pathway

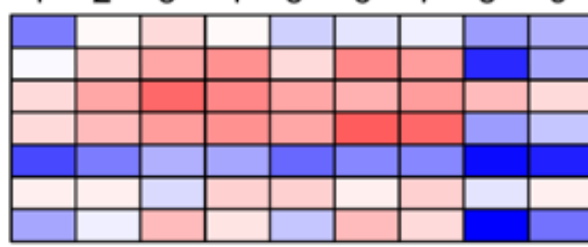

guanine

hypoxanthine

inosine

xanthine

Purine metabolism

xanthosine

sulfuric acid

uric acid

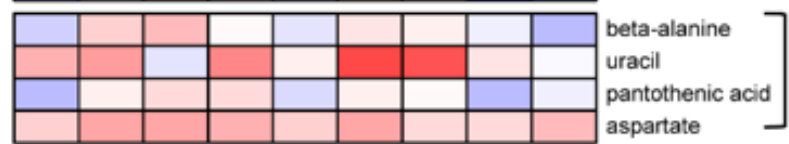

Pantothenate and

CoA biosynthesis
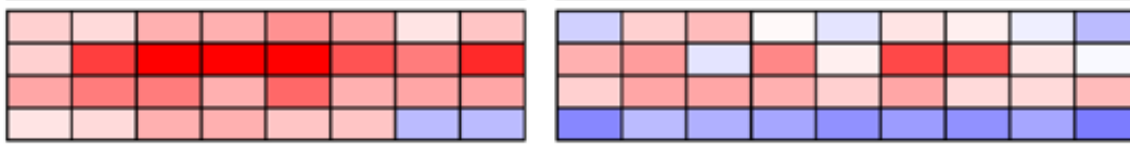

beta-alanine

uracil

aspartate

beta-Alanine metabolism
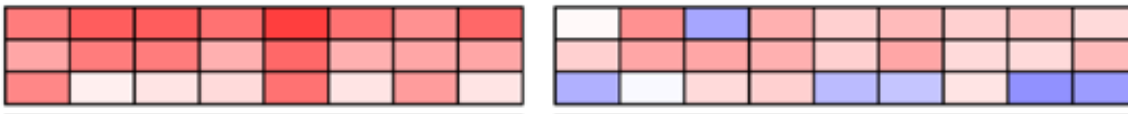

3-hydroxypropionic acid -

glutamic acid

aspartate

glutaric acid

Arginine biosynthesis
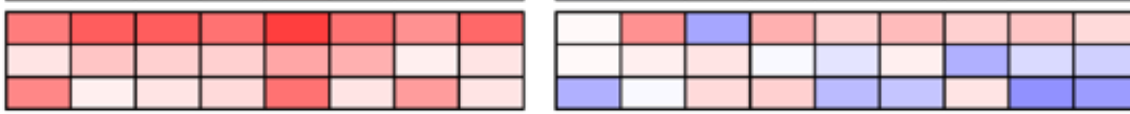

glutamic acid

beta-hydroxybutyric acid

glutaric acid

Butanoate metabolism

$\log _{10}$ (Peak area)

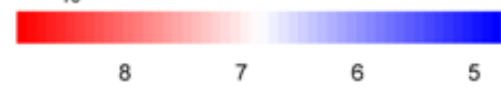

\section{Figure 7}

Key metabolites and pathway enrichment analysis based on metabolomics. (A) Principal component analysis (PCA) score plots for discriminating the faecal metabolome from the control and SenA (100 $\mathrm{mg} / \mathrm{kg}$ ) groups. (B) Disturbed metabolic pathways in the control versus SenA groups. (C) Heatmaps of the differential metabolites that were contained in enriched pathways and were altered by sennoside $A$ $(100 \mathrm{mg} / \mathrm{kg})$ compared with that of control mice $(n=8-9)$. The differences in abundance distributions 
among metabolites between the two groups were measured by GC-MS, and P values less than 0.05 were considered statistically significant. See also Figure S11 and supplementary dataset S1.

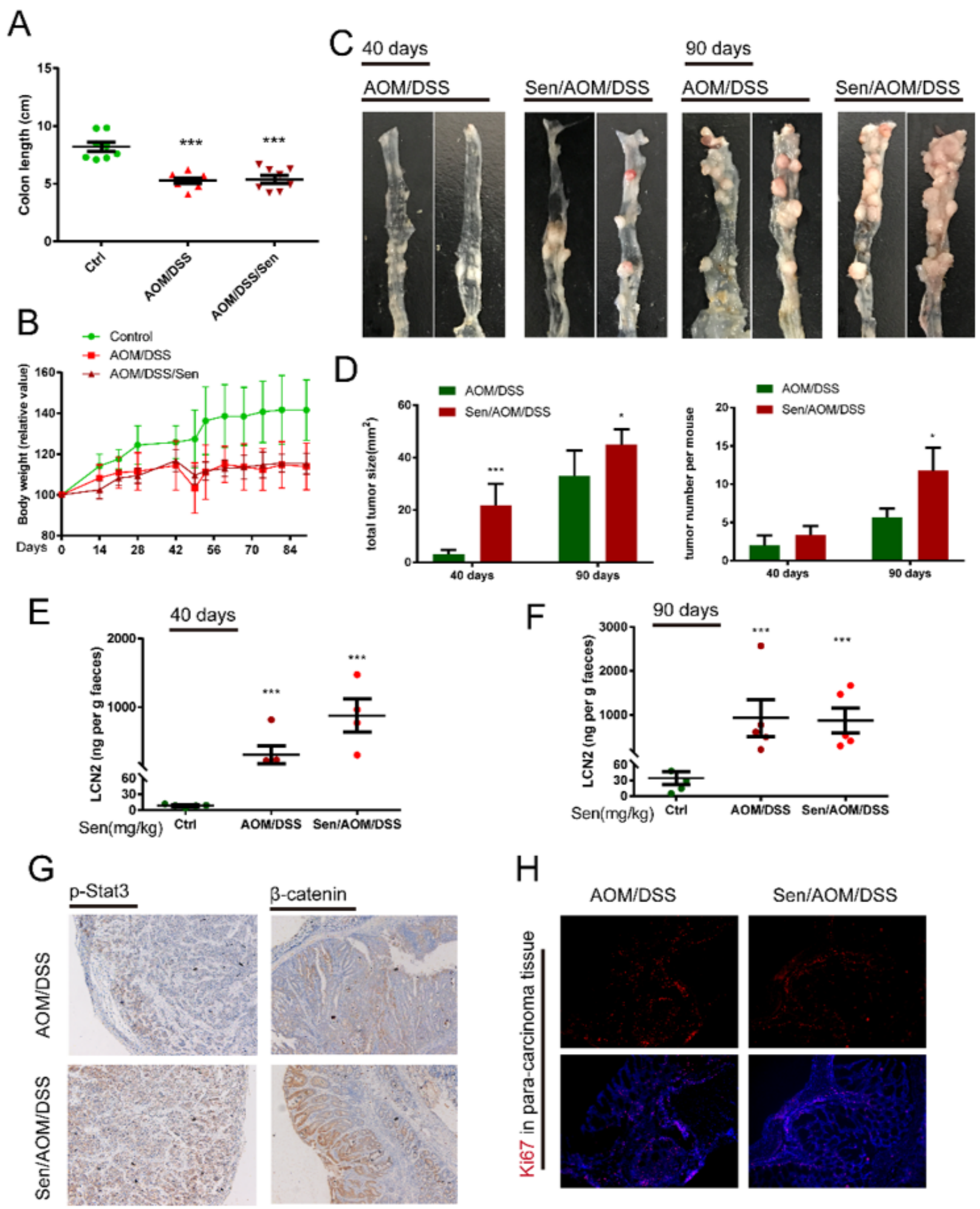

Figure 8

Inflammation-induced tumorigenesis is enhanced in mice treated with sennoside A. (A) Gross morphology and length of the large bowel in different groups. ${ }^{* \star} p<0.001$ versus the control group. (B) Changes in body weight over a period of observation are shown as the percent of the initial weight at the 
start of experiments. (C) Representative image of the colon in mice 40 days and 90 days after the first azoxymethane injection. (D\&E) Size and number of colon tumours. Error bars, mean hSEM. ${ }^{*} p<0.05,{ }^{* *} \mathrm{p}$ $<0.001$ as calculated by t-test. (E\&F) Faecal levels of the inflammatory marker LCN2 at day 40 and day 90 postinjection. (G) Immunohistochemical analysis of phospho-STAT3 and $\beta$-catenin expression in tumours (day 90). (H) Immunofluorescence images for the detection of Ki67 in paracarcinoma tissue.

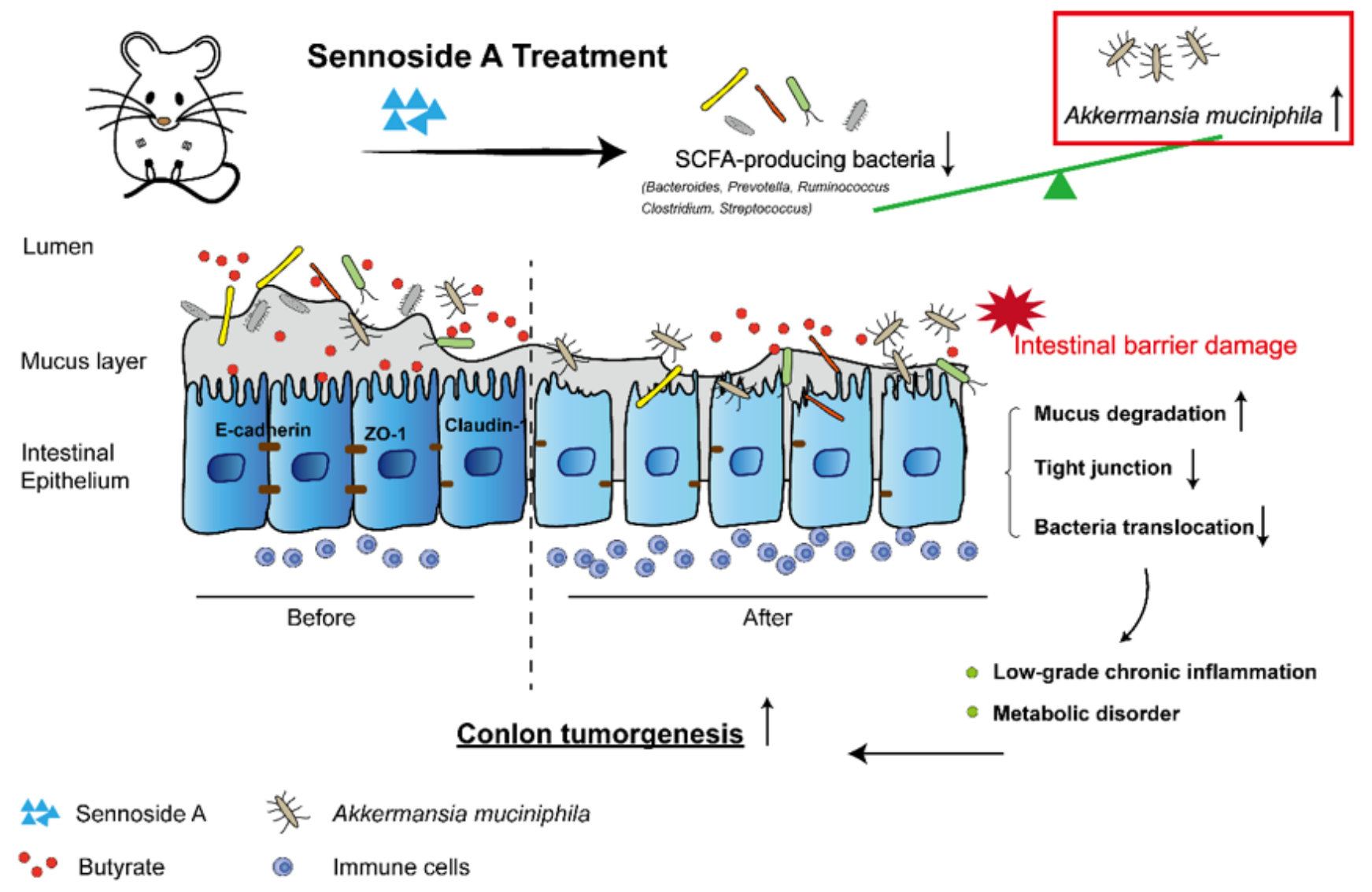

Figure 9

Graphical Abstract. Anthraquinone laxative damages colonic mucosal barrier integrity for colorectal cancer progression by upsetting the balance of intestinal microbial composition.

\section{Supplementary Files}

This is a list of supplementary files associated with this preprint. Click to download.

- supplementarydatasetS1.xlsx

- Sl.docx 